\title{
Aktuelle Behandlungskonzepte für Hirntumoren im Kindes- und Jugendalter
}

Aurelia Peraud, Marie Schuler-Ortoli, Jana Stursberg, Wiebke Schlötzer, Meinrad Beer, Stephan Bartholomä, Mike-Andrew Westhoff, Birgitta Welte, Thomas Wiegel,

Wolfgang Loichinger, Klaus-Michael Debatin

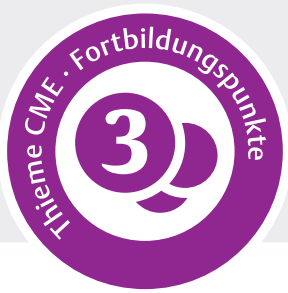

\begin{abstract}
Neue wissenschaftliche Erkenntnisse in der Molekulardiagnostik und Therapie konnten für einige kindliche Hirntumoren erhebliche Verbesserungen für das Gesamt- und rezidivfreie Überleben erzielen. Dennoch ist man sich der Langzeitfolgen der teils sehr invasiven Therapien bei Kindern, der damit verbundenen reduzierten Lebensqualität sowie dem Risiko der Zweitmalignome bewusst. Deshalb ist es wichtig, dass Entscheidungen über das optimale therapeutische Vorgehen für jeden Patienten individuell und interdisziplinär im pädiatrischen Tumorboard getroffen werden.
\end{abstract}

\section{Epidemiologie und Klinik}

Primäre Tumoren des zentralen Nervensystems - also des Gehirns und des Rückenmarks - sind nach den Leukämien die zweithäufigste Krebsentität des Kindesalters und stellen fast $1 / 4$ der dem deutschen Kinderkrebsregister gemeldeten Fälle dar [1]. Die jährliche Inzidenz beträgt etwa 2-4/100000 Kinder im Alter von 0-15 Jahren [2].

Interessanterweise machen im Vergleich dazu primäre ZNS-Tumoren nur 2\% der Krebserkrankungen des Erwachsenen aus; viel häufiger finden sich bei ihnen ZNSMetastasen anderer Neoplasien. Bemerkenswert ist zudem, dass 23\% der Sekundärmalignome nach einer Tumorerkrankung im ZNS zu finden sind.

Über die letzten Jahrzehnte konnte in der Behandlung primärer ZNS-Tumoren (welche je nach Tumorart eine Kombination aus chirurgischer Resektion, Chemotherapie und Bestrahlung ist) eine kontinuierliche Verbesserung des 5Jahres-Überlebens erreicht werden. So beträgt dieses insgesamt derzeit fast $80 \%$ [1]. Allerdings hängt die Prognose des Patienten entscheidend von der Histologie des Tumors und zumeist von der vollständigen Resektabilität ab.

Histologisch werden ZNS-Tumoren gemäß den WHO-Kriterien eingeteilt ( $\mathbf{A} \mathbf{A b} \mathbf{b}$. 1$)$. Am häufigsten finden sich im Kindesalter astrozytäre Tumoren (30-35\%), welche in ihrer Dignität ein Spektrum von niedrig maligne (pilozytische Astrozytome, WHO-Grad I) bis hoch maligne (Glioblastome, WHO-Grad IV) umfassen [2]. Bezüglich ihrer bevorzugten Lokalisation lassen sich diese Tumoren keiner bestimmten Gehirnregion zuordnen. Sie können sowohl supratentoriell, infratentoriell, im Bereich der Seh- bahn als auch im Hirnstamm oder spinal auftreten. Das mittlere Erkrankungsalter ist ebenfalls sehr variabel und erstreckt sich bis in das Erwachsenenalter.

In der Häufigkeit des Auftretens schließen sich mit 20\% die embryonalen Tumoren an. Hierzu gehören u.a. die Medulloblastome, die fast immer in der hinteren Schädelgrube lokalisiert sind. Dort finden sich auch $2 / 3$ der ependymalen Tumoren, die mit 10-15\% ebenfalls zu den häufigeren histologischen Entitäten gehören und eine wichtige Differenzialdiagnose zu den Medulloblastomen dar-

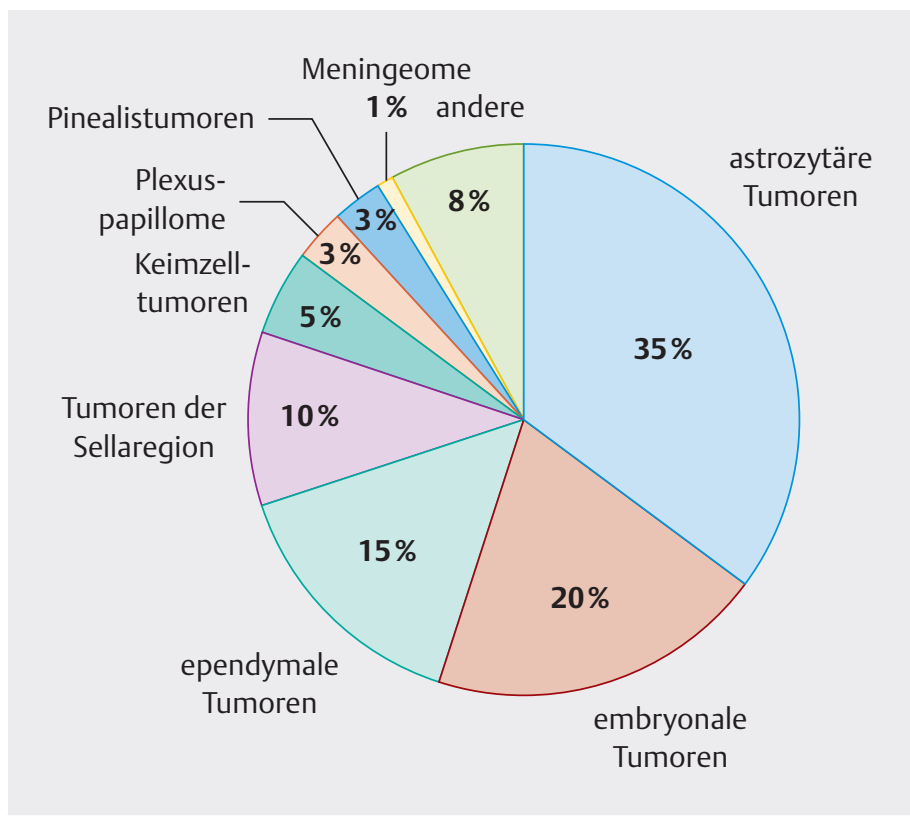

- Abb. 1 Häufigkeitsverteilung kindlicher Hirntumoren. 
stellen. Ihre bevorzugte Lokalisation ist hierbei der IV. Ventrikel, häufig mit Ausbreitung in den Kleinhirnbrückenwinkel und/oder in Richtung Hirnstamm. Sowohl Medulloblastome als auch Ependymome können spinale Abtropfmetastasen bilden. Primär spinale Ependymome (5\% der Ependymome) sind molekularbiologisch und prognostisch als eine eigene Entität anzusehen und betreffen meistens Jugendliche oder Erwachsene [3].

Selten sind Tumoren der Sellaregion (Kraniopharyngeom, Hypophysenadenom, Hypophysenkarzinom [8-10\%]), Keimzelltumoren (Teratome, Germinome, Dottersacktumoren, Chorionkarzinome [3-5\%]), Tumoren des Plexus choroideus [2-3\%], Pinealistumoren [2-3\%] und Meningeome [0-1\%]. Darüber hinaus müssen differenzialdiagnostisch Lymphome und histiozytäre Tumoren in Betracht gezogen werden.

Neue diagnostische Möglichkeiten eröffnen mittlerweile neben der rein histologischen Klassifikation weitere Methoden der Eingruppierung. So können z. B. Medulloblastome genetisch (je nach Aktivierung von WNT und $\mathrm{SHH}$ sowie dem Mutationsstatus des TP53-Gens) in 4 Subgruppen unterteilt werden. Dies ist insofern von Relevanz, als die genetischen Subgruppen mit einer unterschiedlichen Prognose bezüglich des Überlebens der Patienten assoziiert sind, so dass diese in aktuellen Studien einer unterschiedlichen Therapie zugeführt werden [4].

Die klinische Symptomatik der ZNS-Tumoren kann zum einen durch eine Hirndrucksteigerung, zum anderen durch lokalisationsspezifische Störungen der Hirnfunktion hervorgerufen werden. Oft sind die Symptome zunächst unspezifisch, sodass die Diagnosestellung nicht selten verzögert wird.

Ein erhöhter intrakranieller Druck kann sowohl durch das lokale Tumorwachstum als auch durch die Verlegung des Liquorabflusses entstehen. Klinische Zeichen eines erhöhten Hirndrucks sind v.a. Kopfschmerzen und Erbrechen und liegen bei über $50 \%$ der Patienten zum Zeitpunkt der Diagnosestellung vor. Nicht selten bestehen diese Beschwerden seit Wochen oder Monaten, werden jedoch oft zunächst als Ausdruck eines weit häufiger auftretenden Magen-Darm- oder grippalen Infekts interpretiert. Darüber hinaus leiden $10-20 \%$ der Kinder unter chronischen Kopfschmerzen oder einer Migräne - ein Tumor steckt nur in den seltensten Fällen hinter dieser Symptomatik. Folgende Besonderheiten allerdings sollten jeden Kliniker hellhörig werden lassen [2]:

- Zunahme der Kopfschmerzen bei Husten, Niesen oder Pressen

- gestörte Nachtruhe durch Zunahme der Schmerzen im Liegen

- okzipitale Kopfschmerzen

- Nuchalgien

- Nüchternerbrechen

\section{Merke}

Speziell bei Säuglingen kann eine intrakranielle Drucksteigerung anfangs aufgrund der noch offenen Fontanelle und Schädelnähte durch ein vermehrtes Kopfwachstum kompensiert werden. Daher gehören auch eine perzentilenkreuzende Zunahme des Kopfumfangs oder eine vorgewölbte vordere Fontanelle zu den wichtigen Warnsymptomen.

Störungen der Hirnfunktion durch das Tumorwachstum resultieren je nach Lokalisation der Raumforderung in den unterschiedlichsten Symptomen. 45\% der ZNS-Tumoren im Kindesalter sind supratentoriell gelegen und können hier z. B. zu epileptischen Anfällen, aber auch zu Paresen, Gefühlsstörungen, Sehstörungen, Sprachstörungen, endokrinologischen Auffälligkeiten (Wachstumsstörungen, Störungen der Pubertät, Diabetes insipidus oder Schilddrüsenüber- bzw. -unterfunktion) oder psychischen Auffälligkeiten führen. Weitere 52\% der Tumoren sind in der hinteren Schädelgrube lokalisiert. Im Sinne einer Kleinhirn- oder Hirnstammsymptomatik fallen sie u.a. durch Gangstörungen, Schwindel, Singultus, Torticollis oder Hirnnervenausfälle (Doppelbilder, Fazialisparese, Schluckstörung, Heiserkeit) auf [2]. Die insgesamt sehr selten auftretenden primär intraspinalen Tumoren rufen eine (je nach Höhe der Läsion unterschiedlich geartete) Querschnittsymptomatik, z.T. auch mit Blasen-/ Mastdarmstörung, hervor.

Diese fokal-neurologischen Defizite treten oft schleichend auf und werden zudem aufgrund der neuronalen Plastizität im Kleinkindalter teilweise kompensiert, was die Diagnosestellung weiter erschwert. Seltener kommt es - vor allem bei einer akuten Größenprogredienz des Tumors, z.B. im Rahmen einer lokalen Einblutung - zu einem Apoplex. Dies führt meist zu einer notfallmäßigen Klinikeinweisung und Bildgebung mittels Computertomografie. Ansonsten ist zur Sicherung des klinischen Verdachts die Magnetresonanztomografie bildgebende Methode der Wahl.

\section{MRT-Bildgebung}

Die Magnetresonanztomografie (MRT) ist aufgrund des guten Weichteilkontrasts und bei fehlender Strahlenexposition die präferierte Methode in der Hirntumordiagnostik. Die Untersuchung der pädiatrischen Patientengruppe sollte im besonderen Maße standardisiert erfolgen, um eine Reproduzierbarkeit innerhalb von multizentrischen Studien zu gewährleisten. Gängige MRT-Studienprotokolle zur intrakraniellen Tumordiagnostik an einem 1,5- oder 3-Tesla-Scanner umfassen mindestens:

- T1-gewichtete Sequenzen nativ und nach i.v. Gadolinium (3-D-Sequenzen und/oder herkömmliche 2D-Spinechosequenzen, alternativ Gradientenechos)

- eine FLAIR (fluid-attenuated inversion recovery)

- eine T2-gewichtete Spinechosequenz 
- eine diffusionsgewichtete Sequenz (DWI) mit ADCKarte (apparent diffusion coefficient)

Die bevorzugte Raumebene ist axial. Nach Kontrastmittel (KM) sind mindestens 2, zumeist 3 Raumebenen abzubilden [5, 6]. Die Vorteile isotroper 3-D-Sequenzen sind:

- geringe Schichtdicke von $\leq 1,5 \mathrm{~mm}$ mit Rekonstruktionsmöglichkeiten in allen Raumebenen

- bessere Detektion und Reproduzierbarkeit von kleinen Läsionen

- Möglichkeit der Tumorvolumetrie

- Grundlage für die Neuronavigation

Die Protokolle sind an die Erfordernisse der einzelnen Studien (z. B. gemäß den Empfehlungen der European SIOP Brain Tumor Imaging Group) anzupassen.

Die diffusionsgewichtete Bildgebung gehört mittlerweile zum neuroonkologischen Standardprotokoll. Hier wird das MRT-Signal sensitiv für Diffusionseffekte gemacht. Aufgrund der Einschränkung der freien Diffusion von Wassermolekülen durch Zellmembranen existiert ein um- gekehrter Zusammenhang zwischen Tumorzelldichte und der Größe des aus der Diffusionsbildgebung ermittelten scheinbaren Diffusionskoeffizienten ADC. Zellreiche und höhergradige kindliche Hirntumoren (z.B. embryonale Tumoren, höhergradige Gliome) zeigen häufiger einen niedrigen ADC-Wert [7, 12]. Obwohl erhebliche Überlappungen der ADC-Werte für die einzelnen pädiatrischen Tumorentitäten bestehen, ist die diffusionsgewichtete Bildgebung eine hilfreiche Methode. Das gilt u. a. für das häufige differenzialdiagnostische Problem von Neoplasien der hinteren Schädelgrube $[8,9,11]$, wo Medulloblastome regelmäßig eine deutlichere Diffusionsrestriktion als Ependymome oder pilozytische Astrozytome aufweisen ( $\bullet$ Abb. 2).

Im Falle eines Tumors mit hoher Wahrscheinlichkeit für eine meningeale Tumoraussaat (z. B. Medulloblastome) besteht die Notwendigkeit einer spinalen, möglichst präoperativen MRT-Diagnostik. Die spinale Bildgebung kann als Einzeluntersuchung erfolgen oder unmittelbar an die Schädeldiagnostik angefügt werden. So lassen sich eine weitere Narkose und eine 2 . Kontrastmittelgabe vermei-
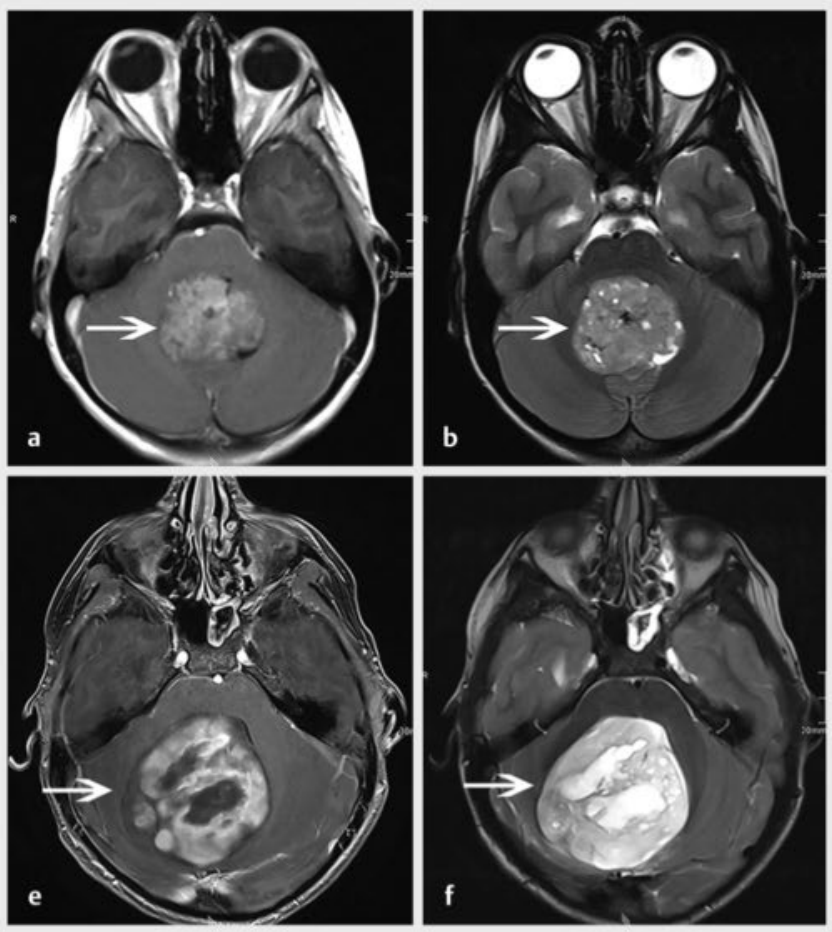
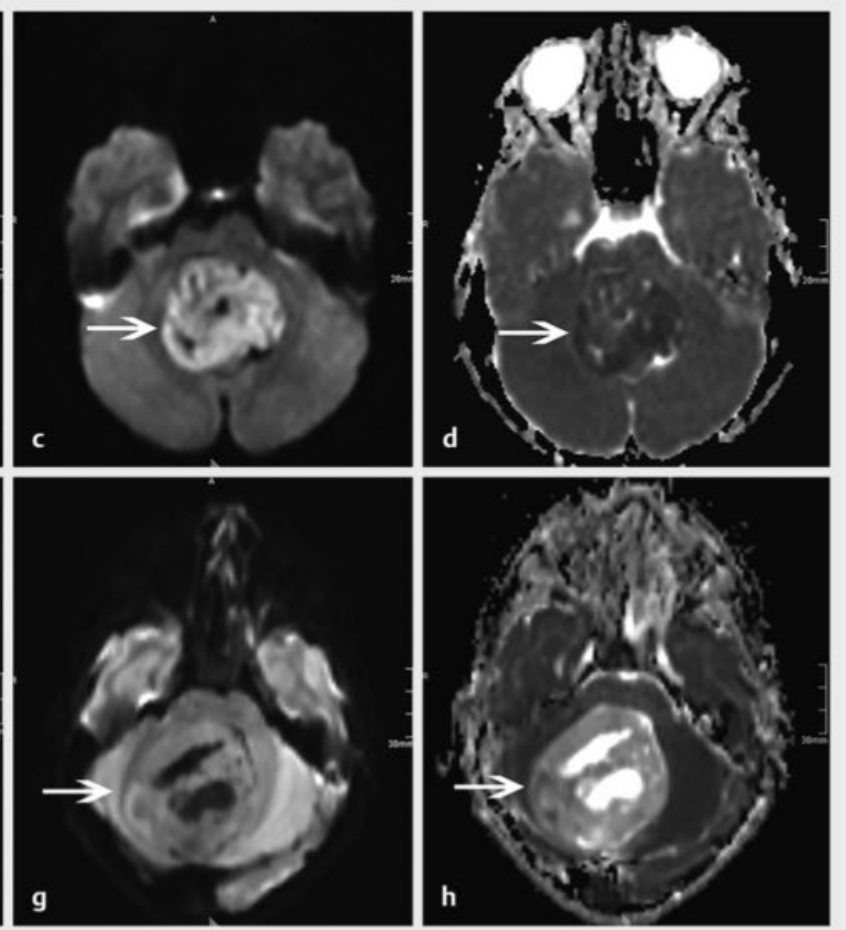

- Abb. 2 MRT des Schädels: Gegenüberstellung Medulloblastom eines 7-jährigen Mädchens (a-d) und pilozytisches Astrozytom bei einem 6-jährigen Jungen (e-h) im 4. Ventrikel. Tumor jeweils mit Pfeilmarkierungen: Das Medulloblastom zeigt in der T1-gewichteten Sequenz eine flaue KM-Aufnahme (a) und ist in der T2-gewichteten TSE-Sequenz gut im 4. Ventrikel ohne Infiltration der Nachbarstrukturen abzugrenzen (b). Entsprechend seiner hohen Zelldichte (und mutmaßlich höheren Malignität) findet sich eine deutliche Diffusionsrestriktion, im DWI-Bild hyperintens/ hell (c), im ADC-Bild entsprechend hypointens/dunkel (d). Das pilozytische Astrozytom imponiert aufgrund seiner Nekrosen im T1-gewichteten Bild nach KM (e) und im T2-gewichteten Bild (f) eher maligne, hat aber in der DWI (g) keinerlei Diffusionsrestriktion: Der Tumor erscheint in seinen soliden Anteilen ähnlich wie das umliegende Hirnparenchym, die zentralen Nekrosen sind dunkel. In der ADC (h) bleibt er entsprechend hyperintens/hell zum umliegenden Gewebe. Damit ergeben sich aus radiologischer Sicht aufgrund der Lage, des Wachstumsverhaltens und der Diffusionsbilder Hinweise auf einen benignen Tumor. 


\section{FALLBEISPIEL}

\section{Pilozytisches Astrozytom WHO-Grad I}

Ein 15-jähriger Junge mit bekannter Zerebralparese stellt sich notfallmäßig wegen seit 2 Monaten anhaltender Kopfschmerzen sowie Nüchternerbrechen vor. Hinzu kam in den letzten 2 Wochen eine Verstärkung der spastischen Gangstörung, welche ihn zuletzt gehunfähig machte. Klinisch neurologisch besteht neben der spastisch-ataktischen Gangstörung eine beidseitige chronische Stauungspapille sowie eine horizontale Blickparese.

Die MRT stellt eine große Raumforderung des 4. Ventrikels dar mit kompletter Verlegung des Ventrikellumens und konsekutivem massiven Hydrozephalus (mit Polkappenbildung). Der Hirnstamm und die Medulla oblongata sind komprimiert und nach ventral verlagert.

Der Tumor weist solide und zystische Anteile auf und nimmt relativ homogen Kontrastmittel auf ( $\triangleright$ Abb. $\mathbf{3}$ ).

Unter intraoperativem elektrophysiologischen Monitoring der langen Bahnen (MEP und SEP) und der Hirnstammfunktionen (Hirnnerven IV-XII) erfolgt die mikroskopisch komplette Entfernung des Tumors. Es zeigt sich eine fokale Infiltration des Tumors in den Hirnstamm auf der linken Seite im Bereich des Fazialiskerngebiets. Der histopathologische Befundbericht belegt ein pilozytisches Astrozytom WHO-Grad I. Eine adjuvante Chemo- oder Radiotherapie war nicht notwendig.

Postoperativ bestehen zunächst ein ausgeprägter Blickrichtungsnystagmus, eine zerebelläre Ataxie sowie eine eingeschränkte Modulation der Stimme bei depressiver Stimmungslage im Sinne eines milden zerebellären Mutismus. Die MRT-Kontrolle belegt die Tumorresektion und die Regredienz des Hydrozephalus.

Unter intensiven Rehamaßnahmen erholt sich der Junge hervorragend. Die Ataxie und die Blickparese sind nach 3 Monaten nicht mehr nachweisbar. Das Gangbild ist sehr viel flüssiger und nur noch angedeutet spastisch-ataktisch. Vom Affekt ist der junge Mann nicht mehr depressiv und sehr viel interaktiver als zuvor.

In den MRT-Verlaufskontrollen sieht man allerdings eine langsam progrediente Zunahme einer residuellen Kontrastmittelaufnahme im Bereich der vormaligen Tumoransatzstelle, sodass die Reoperation im Rahmen der Tumorboard-Diskussion als Alternative zu anderen therapeutischen Optionen wie einer fokalen Bestrahlung oder einer Chemotherapie angeboten wurde.
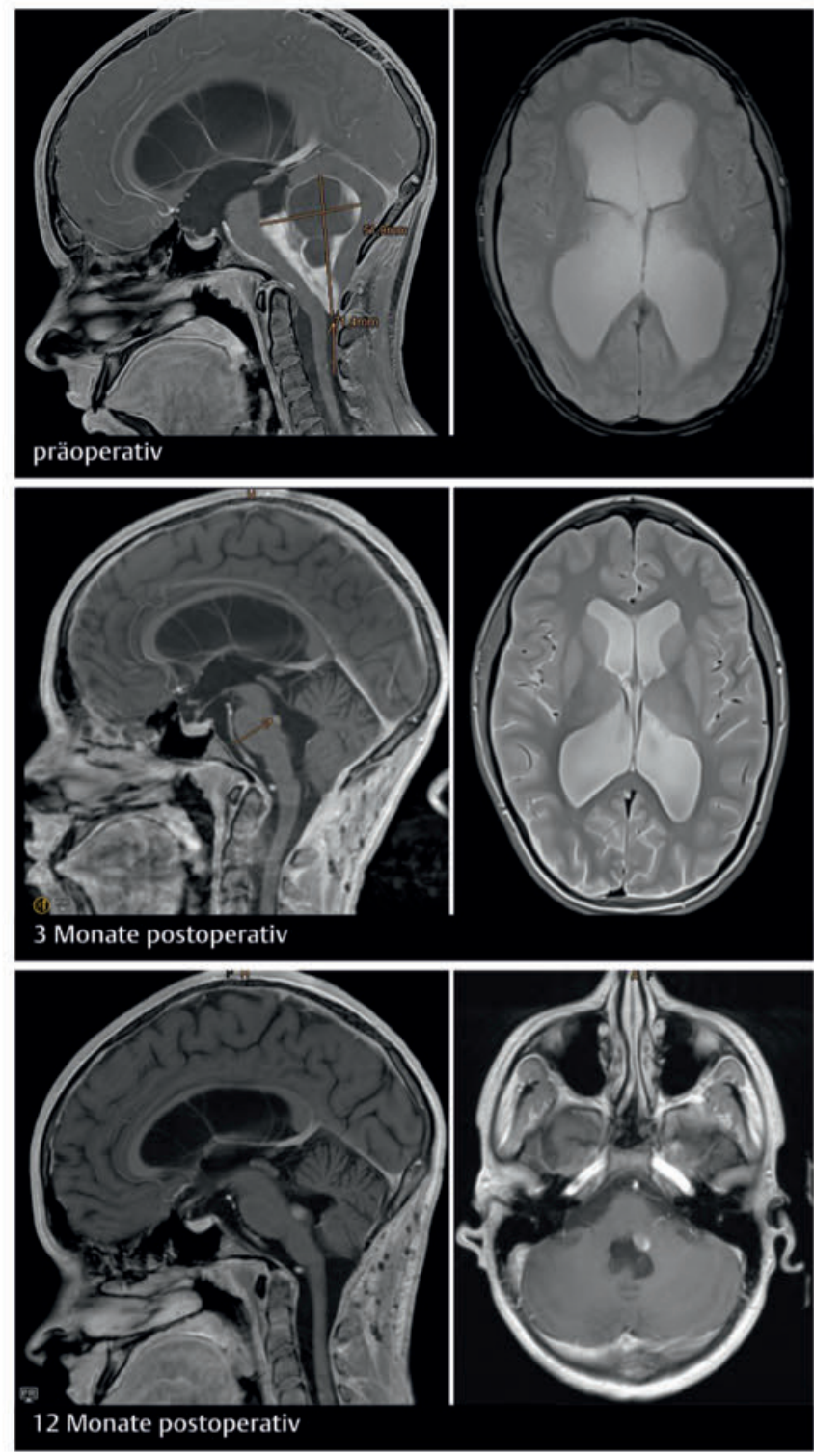

- Abb. 3 MRT. Pilozytisches Astrozytom WHO-Grad I eines 15-jährigen Jungen. 
den. Ein pragmatisches Vorgehen wäre es hier, auf die native Untersuchung zu verzichten und den gesamten Spinalkanal ausschließlich mit T1-gewichteten Sequenzen nach KM in sagittaler und transversaler Schnittführung darzustellen. T2-gewichtete Sequenzen, im Speziellen solche mit einem hohen Liquor-Gewebe-Kontrast (z. B. CISS, FIESTA-C, b-FFE o. ä.), können sinnvoll zur Detektion von kleinen knotigen Auflagerungen auf den intraduralen Spinalnerven ergänzt werden. Ist das spinale Basis-MRT nicht präoperativ erfolgt, ist dieses innerhalb von 72 Stunden postoperativ nachzuholen. Sollten hier ausgedehnte subdurale Effusionen und diagnostische Unklarheiten auftreten, kann eine frühe Wiederholung der Untersuchung nach 2-3 Wochen notwendig werden [5].

Sogenannte „subdurale Effusionen“ werden auch als „unspezifisches subdurales Enhancement“ oder "postoperative intraspinale subdurale Kollektionen" bezeichnet. Sie treten gehäuft nach Eingriffen in der hinteren Schädelgrube auf und zeigen i.d.R. eine vollständige Rückbildung innerhalb von wenigen Wochen. Sie sind klinisch asymptomatisch [10]. Aufgrund des typischen MRTAspekts mit lobulierten intraspinalen Formationen, die zwar homogen KM aufnehmen, in den T2-gewichteten Sequenzen aber liquorisointens erscheinen, sollten sie nicht mit Metastasen zu verwechseln sein ( $\mathbf{A b b} \mathbf{4} \mathbf{4}$, Abb.5). Sie können jedoch die Diagnostik einer intraspinalen Tumoraussaat erschweren [14].

Das postoperative Schädel-MRT dient der Statuserhebung und Dokumentation eines Resttumors. Es sollte innerhalb von 24-48, max. 72 Stunden durchgeführt werden.

\section{Merke}

Da die Verwendung eines lokalen Hämostyptikums oder von zytostatikahaltigen Implantaten erfahrungsgemäß nicht unerhebliche postoperative Veränderungen nach sich ziehen, muss dieses bei der Interpretation der Bilder berücksichtigt werden.

Bei diagnostischen Untersicherheiten ist eine kurzfristige weitere MRT-Kontrolle angezeigt. Verlaufskontrollen werden den verschiedenen Nachsorgeplänen für die jeweiligen Tumorentitäten angepasst und sollten für eine valide Vergleichbarkeit identisch zu den Voruntersuchungen sein [13].

Während bei kleinen Kindern mit regulären onkologischen MRT-Untersuchungen aufgrund der Länge der erforderlichen Protokolle zumeist eine Narkose notwendig ist, stehen für klinische Fragestellungen (z. B. Ventrikelweite) sehr schnelle Sequenzen zur Verfügung, die auch bei dieser Patientengruppe eine wache Untersuchung mit ausreichender diagnostischer Aussagekraft zulassen.

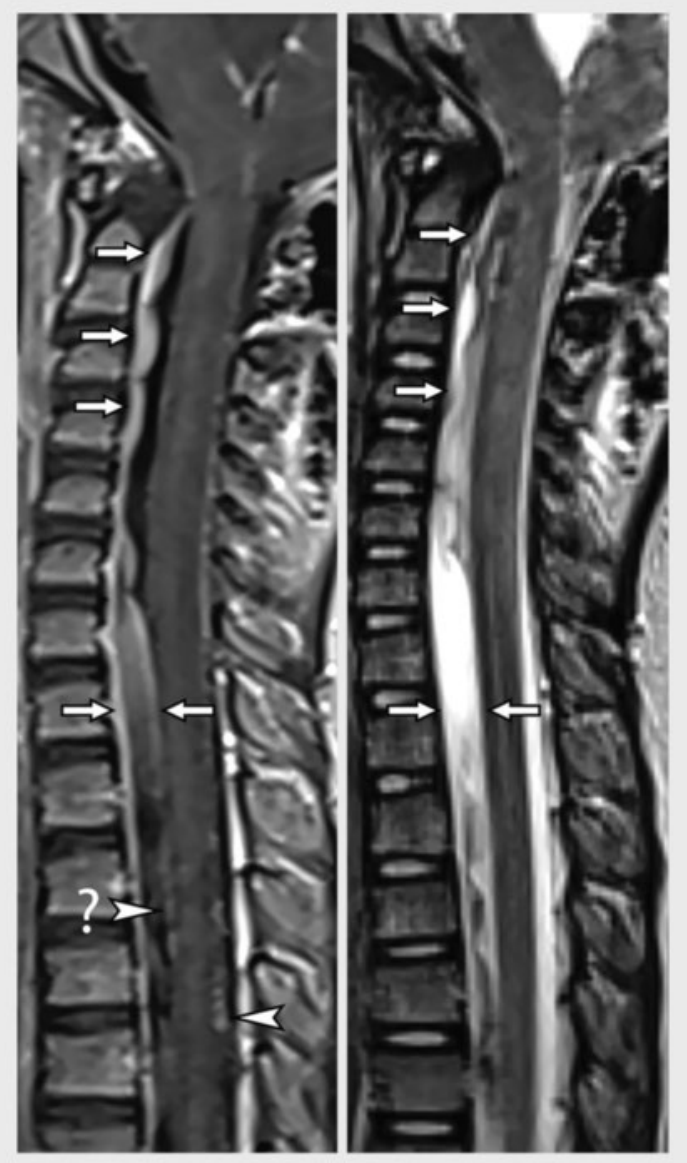

- Abb. 4 Spinales MRT. Subdurale Effusionen, 2-jähriges Mädchen unmittelbar postoperativ nach kompletter Resektion eines anaplastischen Ependymons aus der hinteren Schädelgrube. Links: Bei den mit Pfeilen markierten KM-aufnehmenden konvexen Befunden innerhalb des Spinalkanals handelt es sich um postoperative Veränderungen ohne Krankheitswert (T1-TSE HWS/BWS nach Gadolinium). Allerdings kann es in dieser Sequenz schwer sein, kleine KM-Aufnahmen auf der Oberfläche des Myelons (Pfeilspitzen) von einer spinalen Aussaat zu differenzieren. Rechts: Die subduralen Effusionen zeigen in der T2-gewichteten TSE-Sequenz ein ähnliches hyperintenses/ helles Signal wie Liquor. Alle intraspinalen Auffälligkeiten hatten sich in der Kontrolle nach 4 Monaten komplett rückgebildet (ohne Abbildung). Derartige diagnostische Unsicherheiten lassen sich durch Anfertigung des spinalen MRTs bereits präoperativ vermeiden.

Über die Basisdiagnostik hinaus bietet das MRT-Portfolio zahlreiche weitere Methoden zur Klärung klinischer Fragen. Hierzu zählen die MR-Angiografie, -Spektroskopie, -Perfusion, die funktionelle Kernspintomografie (FMRI) zur Klärung der Lagebeziehung des Tumors zu eloquenten Arealen sowie die suszeptibilitätsgewichtete MRT (SWI) zur Abbildung der Mikrovaskularisation, von Blutbestandteilen oder Verkalkungen. Im stetigen Bemühen um Komfort, Sicherheit und diagnostische Genauigkeit 


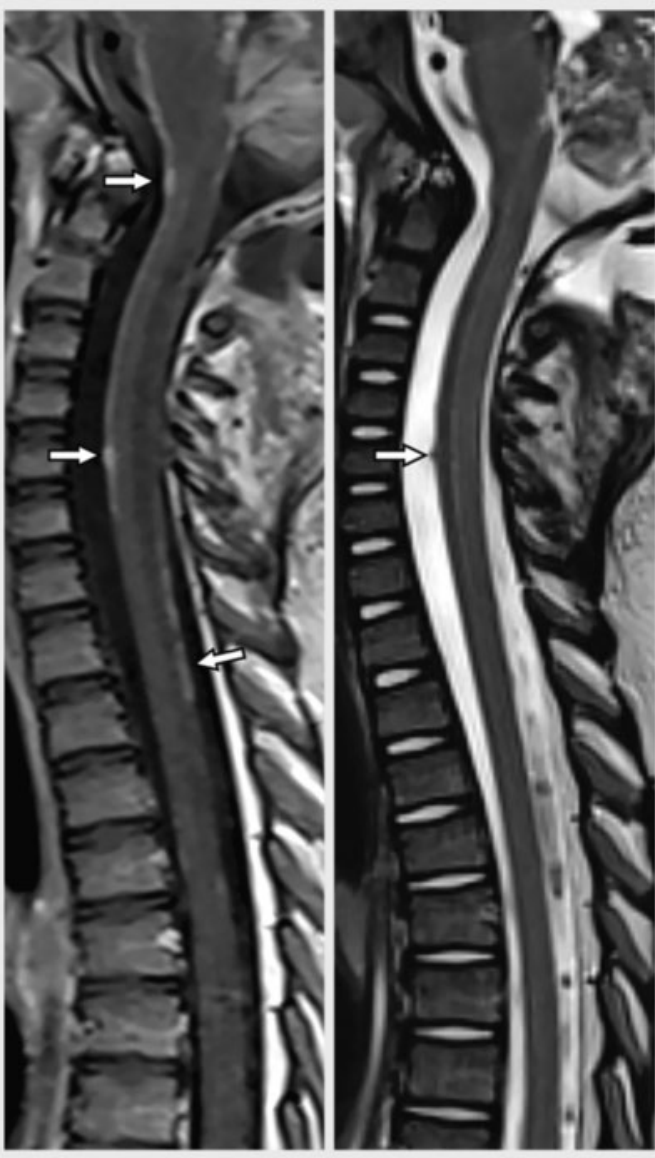

- Abb. 5 Spinales MRT. Meningeale Aussaat bei einem 2-jährigen Jungen, Z.n. Medulloblastomresektion aus dem 4. Ventrikel. Links: Das Myelon ist zuckergussartig, z. T. nodulär (exemplarische Pfeile), mit KM-aufnehmenden Zellverbänden überzogen (T1-TSE HWS/BWS nach Gadolinium). Rechts: In T2 sind diese Veränderungen schlechter nachzuvollziehen. Ein hier markierter Tumorknoten ist hypointens/dunkel abzugrenzen.

für die pädiatrische Patientengruppe sind die Weiterentwicklung schnellerer und leiserer Sequenzen, kontrastmittelfreie Methoden (z.B. MRT-Perfusion mittels ASL = arterial spin labeling) oder Verfahren, die potenziell molekulare Tumorveränderungen detektieren können (z.B. CEST = chemical exchange saturation transfer) zukunftsträchtige Ansätze.

Weitere Perspektiven ergeben sich durch Analyse des Bildmaterials durch Verfahren der künstlichen Intelligenz, die mehr als der menschliche Befunder in der Lage sind, quantitative Informationen aus den Bildern zu extrahieren und in den Kontext histologischer und molekularer Tumoreigenschaften oder Prognosenparameter zu stellen („radiomics“). So könnte es in Zukunft weitaus besser möglich sein, Tumoren anhand ihrer Bildeigenschaften zu klassifizieren oder Aussagen über den möglichen Verlauf und das Therapieansprechen zu machen.

\section{Neurochirurgische Therapie}

Der chirurgischen Tumorentfernung kommt in der Therapie von kindlichen ZNS-Tumoren nach wie vor ein hoher Stellenwert zu, sofern diese mit einer niedrigen Morbidität möglich ist. Dies erfordert eine präzise präoperative Planung basierend auf hochauflösenden MRT-Bilddatensätzen, wenn notwendig moderne Neuronavigationsverfahren und den Einsatz von intraoperativer Elektrophysiologie zur funktionellen Überwachung während des Eingriffs. Neben der Reduktion der Tumorlast und der möglichen Verbesserung von Symptomen durch die mikrochirurgische Resektion kommt der histologischen und molekularen Diagnosesicherung eine wichtige Bedeutung zu.

\section{Merke \\ Ist aufgrund der hocheloquenten Lage eine Resektion nicht gefahrenarm möglich, sollte der stereotakti- schen oder navigierten Biopsie der Vorzug gegeben werden.}

Diese Verfahren ermöglichen nicht nur hochpräzise die Entnahme von Tumorproben, sondern vermögen auch tumorassoziierte Zysten zu drainieren oder einem kompressionsbedingten Hydrozephalus durch die Anlage eines inneren Shunts zu begegnen.

Zunehmende Erkenntnisse über molekulare Signaturen in kindlichen ZNS-Tumoren und deren potenzielle prognostische Bedeutung machen eine bioptische Tumorprobenentnahme auch bei Hirnstammgliomen mehr und mehr notwendig. Dies wurde in der Vergangenheit nicht einheitlich gehandhabt und viele Kinder wurden ohne histologische Diagnosesicherung einer Chemotherapie oder Bestrahlung zugeführt.

Die Tatsache, dass Tumoren, die nach gängigen histologischen Kriterien einer Tumorentität angehören, dennoch gänzlich unterschiedliche molekulare Veränderungen aufweisen können und sich auch biologisch verschieden verhalten, verdeutlicht den Paradigmenwechsel, der sich gerade in der Diagnostik und Therapie von kindlichen ZNS-Tumoren vollzieht. Dies hat sich nicht nur in der neuen WHO-Klassifikation der ZNS-Tumoren sondern auch in Studienprotokollen niedergeschlagen.

\section{Mikrochirurgische Resektion und stereotaktische bzw. navigierte Biopsie}

Moderne Operationsmikroskope ermöglichen es heute, den intraoperativen Situs nicht nur in unterschiedlicher Vergrößerung darzustellen, sondern diesen auch für die Erkennung von pathologischem Gewebe optimal auszuleuchten. Zusätzlich können Gewebe nach Fluoreszenzmarkierung durch spezielle UV-Filter angefärbt und 
so vom normalen Gewebe unterschieden werden. Weitere Optionen bestehen darin, die Navigationsbildgebung sowie Ergebnisse aus elektrophysiologischen Ableitungen in das Operationsbild des Operateurs zu integrieren. Damit wird der Neurochirurg multimodal und kontinuierlich mit allen notwendigen anatomischen, physiologischen und pathologischen Informationen während des Eingriffs versorgt, um für jeden individuellen Patienten ein optimales Resektionsergebnis erzielen zu können.

Ziel des operativen Eingriffs bei kindlichen Hirntumoren ist es, eine weitgehend komplette Resektion des Tumors durchzuführen, sofern dies ohne allzu großes Risiko für zusätzliche neurologische Ausfallerscheinungen möglich ist. Durch die Entfernung der Raumforderung soll zusätzlich eine Symptomkontrolle bzw. -verbesserung erreicht werden. Und nicht zuletzt ermöglicht erst die Entnahme des Gewebes eine weitergehende histologische und molekulare Aufarbeitung des Tumors durch den Neuropathologen. Die Ergebnisse dieser Untersuchungen sind Grundlage für die prognostische Einschätzung und die Entscheidung über weitere adjuvante Therapieverfahren [15-17].

\section{Merke}

Für die meisten kindlichen Hirntumoren ist die Radikalität der Tumorentfernung von wichtiger prognostischer Bedeutung und korreliert signifikant mit der rezidivfreien Zeit und ggf. dem Gesamtüberleben.

Um dieses Ziel auch in kritischen Regionen des Gehirns oder des Rückenmarks zu realisieren, sollte ein intraoperatives neurophysiologisches Monitoring zur Überwachung von motorischen und sensiblen Bahnsystemen sowie Hirnstamm- bzw. Hirnnervenfunktionen routinemäßig zum Einsatz kommen. Der Erhalt der Lebensqualität und damit die Integrität der neurologischen Funktionen des Kindes stehen auch bei der chirurgischen Therapie im Vordergrund. So kann in Einzelfällen nur eine Teilentfernung des Tumors erreicht werden. Zusätzlich erleichtert die Anwendung der Neuronavigation basierend auf einem 3-D-MRT-Datensatz die gezielte Planung und sorgfältige Resektion, gerade bei supratentorieller Tumorlokalisation. Die präoperativen Bildbefunde werden zusätzlich intraoperativ durch kontinuierliche Ultraschalluntersuchungen verifiziert und während der Resektion aktualisiert [18].

Sofern der Tumor in hocheloquenten oder tiefsitzenden Arealen wie den Stammganglien, der Pinealisregion (vor allem wenn V.a. einen Keimzelltumor besteht) oder im Hirnstamm lokalisiert ist, würde man der stereotaktischen oder navigierten Probeentnahme den Vorzug geben.

Mehr noch als bei erwachsenen Patienten ist für die Planung und Durchführung der Operation auf Besonderhei- ten des kindlichen Organismus zu achten. Säuglinge und Kleinkinder kühlen nicht nur relativ rasch aus, was sich auf die Homöostase bei der OP auswirken kann, sondern sie tolerieren in Abhängigkeit vom vorhandenen Blutvolumen oft nur relativ begrenzte Blutverluste, bevor es zu signifikanten Kreislaufstörungen kommt. Ein erfahrenes anästhesiologisches Team ist für die Operation bei Kindern unter 5 Jahren deshalb besonders wichtig. Auch die Lagerung und Fixierung des Kopfes für die Operation kann bei den kleinen Patienten sehr aufwendig sein. Nicht zuletzt erfordert der Umgang mit kindlichem Gewebe und der Einsatz der idealen Operationstechnik bei Kindern Erfahrung.

\section{Onkologische Therapie und molekulare Diagnostik}

Chemotherapie wird nicht nur zur Verbesserung der Überlebensdauer eingesetzt, sondern auch zur Verminderung der Morbidität, vor allem zur Reduktion der Bestrahlungsdosis. Gerade bei Säuglingen und Kleinkindern ist die Chemotherapie die Therapie der 1. Wahl, da eine Bestrahlung des sich noch entwickelnden Gehirns negative kognitive sowie endokrinologische Langzeitfolgen hat.

Die medikamentöse Therapie besteht sowohl aus systemisch wie auch intrathekal bzw. intraventrikulär (Ommaya-Reservoir) applizierter Chemotherapie. Jede der Tumorentitäten zeigt ein unterschiedliches Ansprechen auf die verschiedenen Chemotherapieschemata. Die BlutHirn- bzw. Blut-Liquor-Schranke spielt dabei ebenfalls eine große Rolle. Viele Hirntumoren haben außerdem eine geringe mitotische Aktivität, sodass nur ein kleiner Anteil der Tumorzellen auf Chemotherapie empfindlich ist. Um ausreichende Konzentrationen im Gehirn und Liquor zu erreichen, besteht bei manchen Tumoren die Möglichkeit einer Dosiseskalation durch hochdosierte Chemotherapie mit autologem Stammzellsupport.

Für fast alle kindlichen Hirntumoren existieren spezifische Studienprotokolle der GPOH (Gesellschaft für Pädiatrische Onkologie und Hämatologie; www.kinderkrebsinfo. de/e2260/index_ger.html).

\section{Merke \\ Die Patienten sollten immer in Zentren gemäß den aktuellen Studienprotokollen behandelt werden.}

Aktuell befinden wir uns in einer Umbruchphase, da einige Studien vor Kurzem geendet haben und nun die Folgestudien, aufgrund der in den Vorgängerstudien gewonnenen Erkenntnisse, entweder gerade initiiert worden sind oder sich in Entwicklung befinden. In den Fällen, in denen aktuell keine Studie mit Therapieoptimierungsprotokoll existiert, tritt an diese Stelle ein Register, in dem in 


\section{FALLBEISPIEL}

\section{Glioblastoma multiforme WHO-Grad IV}

Drei Monate alter weiblicher Säugling mit fokalen epileptischen Anfällen der rechten Körperseite und progredientem Makrozephalus. Bei Aufnahme ist das Mädchen wach, aber irritabel mit einem weit über der 97. Perzentile liegenden Kopfumfang und prall gefüllter vorderer Fontanelle. Das Kind weist eine latente Hemiparese rechts auf mit vermehrtem Fäusteln der rechten Hand.

Im MRT zeigt sich eine ausgedehnte supratentorielle, überwiegend linkshemisphärische (frontal, parietal und temporal) heterogene Raumforderung mit diffusionsgestörten kontrastmittelaffinen soliden Anteilen und multiplen zystischen, teilweise eingebluteten Anteilen, hierbei Einblutungen unterschiedlichen Alters. Es besteht eine Okklusion der Seitenventrikel mit Erweiterung insbesondere des linken Temporalhorns und des Hinterhorns ( $>$ Abb. 6).

Es erfolgt eine ausgedehnte Tumorteilresektion, wobei die Anteile im Thalamus auf beiden Seiten belassen werden. Der neuropathologische Befundbericht lautet Glioblastoma multiforme WHO-Grad IV. Dieser Befund wurde auch vom Tumorreferenzzentrum in Bonn bestätigt. Die molekulare Aufarbeitung des Tumorpräparats weist für das IDH1- und IDH2-Gen eine Wildtypsequenz auf. Die 1 p/19 qFISH-Analyse belegt keinen signifikanten Verlust der Heterozygotie auf beiden Allelen. Die Promotorregion des MGMT-Gens ist nicht methyliert.

Die MR-Kontrolle zeigt den Tumorrest im Thalamus beidseits mit intratumoraler Hämorrhagie und eine progrediente Erweiterung der inneren und äußeren Liquorräume.

Initial wird notfallmäßig eine subdurale Drainage links bei zunehmenden Liquorkissen eingelegt. Nach klinischer Stabilisierung erfolgte die Anlage eines Ventrikulo- und subduroperitonealen Shunt mit ProGAV-Ventil.

Nachdem sich das Kind klinisch gut stabilisiert hat und sich auch die Motorik der rechten Körperseite auf das Ausgangsniveau erholt hat, wird eine Polychemotherapie nach dem HIT-SKK-Protokoll initiiert. Aufgrund des jungen Alters des Kindes kommt eine Strahlentherapie nicht in Betracht.

Unter Chemotherapie zeigt der Tumor über 6 Monate zunächst ein gemischtes Ansprechen. Die eingebluteten Tumoranteile im Thalamus werden sukzessive kleiner. Im Anschluss an die Chemotherapie nach dem HIT-SKK-Protokoll erhält das Kind seit 6 Monaten Chemotherapie nach dem RIST-Protokoll (Sirolimus, Irinotecan, Dasatinib, Temozolomid), welches sie gut verträgt. Final wird eine Resektion des verbliebenen Tumorrestes angestrebt.

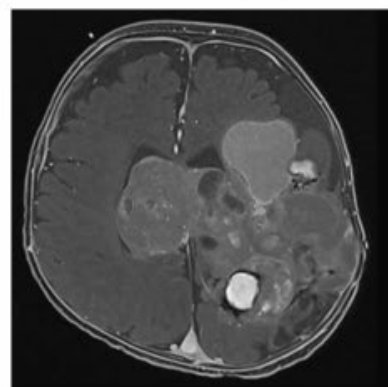

präoperativ

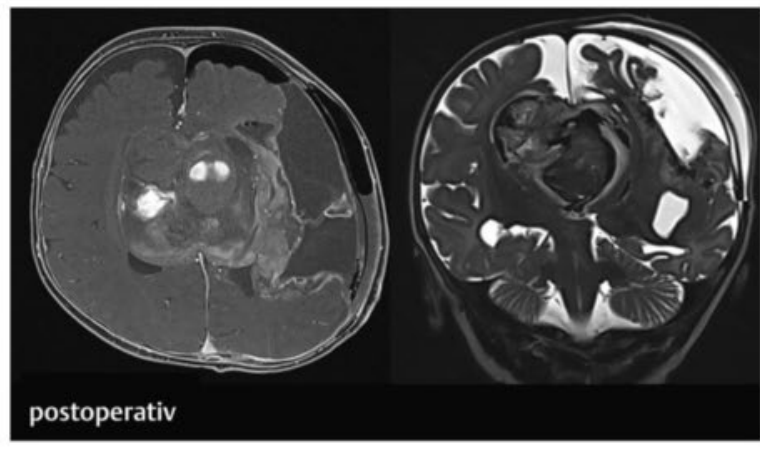

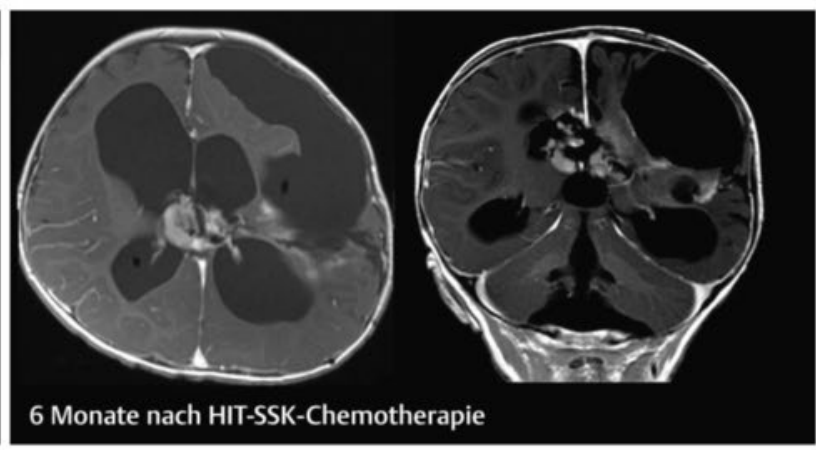

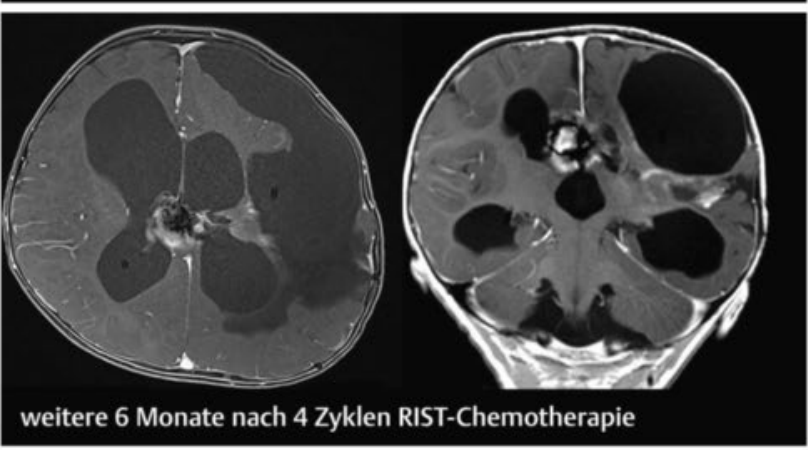

- Abb. 6 MRT. Glioblastoma multiforme WHO-Grad IV eines 3 Monate alten Säuglings. 
der Interimszeit epidemiologische Daten sowie Gewebematerial befundet und gesammelt werden. Den Registern hängen i.d.R. die aktuellen Therapieempfehlungen an, die jedoch nicht bindend sind - die Studienzentralen stehen für Rückfragen bei Diagnostik und Therapieentscheidungen weiterhin zur Verfügung.

Im Folgenden sollen kurz die aktuellen Studienprotokolle genannt bzw. ein Ausblick auf die sich in Entwicklung befindlichen Protokolle gegeben werden. Für genaue und aktuelle Therapieempfehlungen verweisen wir ausdrücklich auf die jeweils aktuellen Studienprotokolle der $\mathrm{GPOH}$.

\section{Behandlung der „Low-Grade-Gliome“}

SIOP Low Grade Glioma 2004 (seit 2012 als Register) und LOGGIC Core-Plattform/LOGGIC Europa-Studie

Mit 30-40\% aller Hirntumoren des Kindesalters stellen niedermaligne Gliome („low-grade glioma“, LGG) die größte histologische Gruppe dar. Im SIOP-LGG-2004-Register und zukünftig in der LOGGIC-Europa-Studie wird der Terminus „Gliom“ von niedrigem Malignitätsgrad benutzt, um alle histopathologisch benignen oder semibenignen glialen und glioneuronalen Tumoren zu bezeichnen, mit Ausnahme diffus intrinsischer Ponsgliome, maligner (anaplastischer) Gliome (WHO-Grad III) und des Glioblastoma multiforme.

\section{Behandlung der Medulloblastome und der primitiven neuroektodermalen Tumoren (PNET)}

HIT-Med-1-Register (GPOH), SIOP PNET 5 MB $(\mathrm{GPOH})$ und HIT-Rez-Register (GPOH)

Primitive neuroektodermale Tumoren gehören zu den embryonalen Tumoren und sind mit 25\% die zweithäufigste Entität aller Hirntumoren bei Kindern und Jugendlichen. Zu etwa 75\% gehen sie vom Kleinhirn aus und werden dann als Medulloblastom bezeichnet. Supratentorielle PNET entstehen überwiegend in den Großhirnhemisphären oder in der Pinealisregion (entsprechende Bezeichnung: Pineoblastom). Bei V.a. ein Medulloblastom/PNET sind neben dem Schädel-MRT immer ein spinales MRT und eine Liquordiagnostik notwendig, da bei primärer Metastasierung eine präoperative systemische Chemotherapie erwogen werden kann. Eine okkulte Metastasierung durch Dissemination von Tumorzellen über den Liquorweg kann initial bei 25\% der Kinder im Liquor nachgewiesen werden.

\section{Behandlung der Ependymome}

SIOP Ependymoma II (GPOH) und HIT Med 1 Register (GPOH)

Ependymome machen 8-12\% aller ZNS-Tumoren im Kindesalter aus, bei Kindern unter 5 Jahren sind es etwa 50\%. Eine Metastasierung entlang des Spinalkanals besteht bei 3-17\% der Patienten zum Zeitpunkt der Diagnosestellung. Bei einer kompletten neurochirurgischen Resektion liegt die 10 -jahres-Überlebensrate bei $70 \%$, während bei inkompletter Resektion die Rate bei 32\% liegt. Somit ist die Bedeutung der Operation klar belegt. Alle Patienten mit Resttumor erhalten eine adjuvante Chemotherapie mit anschließender Bestrahlung.

\section{Behandlung der Rezidive von Medullo- blastomen, Pineoblastomen, primitiven neuroektodermalen Tumoren des ZNS und Ependymomen}

HIT-REZ-Register (GPOH)

In dieses multizentrische Register können Kinder, Jugendliche und junge Erwachsene mit therapierefraktären oder rezidivierten Medulloblastomen, Pineoblastomen, primitiven neuroektodermalen Tumoren des ZNS und Ependymomen aufgenommen werden.

Parallel zu der Registerstudie können in der HIT-REZ-Studienzentrale Therapieempfehlungen eingeholt werde, die auf den aktuellen Ergebnissen nationaler (HIT-REZ97-Studie und HIT-REZ-2005-Studie) und internationaler Rezidivstudien basieren.

\section{Behandlung der intrazerebralen Keimzelltumoren}

SIOP CNS GCT II (GPOH)

Intrazerebrale Keimzelltumoren treten meist mittelliniennah auf, d. h. im Bereich der Pinealis oder in der suprasellären Region. Manchmal werden auch bifokale Tumoren im Bereich der Pinealis und der Hypophyse gefunden. Es handelt sich um die Tumorentitäten der Germinome, der malignen Non-Germinome und der Teratome. Zur initialen Diagnostik gehören neben MRT des gesamten ZNS auch eine Liquoruntersuchung hinsichtlich Zytologie und der Tumormarker AFP und $\beta$-HCG. Zeitgleich müssen die Tumormarker auch im Serum untersucht werden. Bei AFP $>25 \mu \mathrm{g} / \mathrm{dl}$ und $\beta$-HCG $>50 \mathrm{IU} / \mathrm{I}$ kann bei Ausschluss anderer Ursachen und charakteristischer Tumorlokalisation ein sezernierender Keimzelltumor diagnostiziert werden, ohne eine primäre Biopsie durchzuführen.

\section{Behandlung der Kraniopharyngeome}

\section{Kraniopharyngeom 2007}

Bei Kraniopharyngeomen handelt es sich um dysontogenetische Mittellinientumoren. Im Rahmen der retrospektiven Querschnittstudie HIT Endo zeigte sich eine hohe Gesamtüberlebensrate von 92\%, bei jedoch eingeschränkter Lebensqualität und funktioneller Kapazität bei Vorliegen einer hypothalamischen Beteiligung des Kraniopharyngeoms. Eine Hypothalamusbeteiligung war mit Essstörungen, einer ausgeprägten Adipositas, einer hohen Rate an neurochirurgischen Eingriffen und Spätmorbiditäten assoziiert.

Die multizentrische Beobachtungsstudie Kraniopharyngeom 2000 ergab neben den beschriebenen Folgen einer Hypothalamusbeteiligung auch folgende Ergebnisse: 
- Ein radikales operatives Vorgehen wird bei Hypothalamusbeteiligung in Anbetracht der daraus resultierenden Spätfolgen nicht empfohlen.

- Eine komplette Resektion gelingt in ca. 50\% der Fälle.

- Prospektive Untersuchungen zum Zeitpunkt der Strahlentherapie nach inkompletter Resektion eines Kraniopharyngeoms existieren nicht.

\section{Behandlung der hochmalignen Gliome, diffus intrinsischen Ponsgliome und Gliomatosis cerebris}

\section{HIT HGG 2007 und HIT HGG 2013}

Die hoch malignen Gliome, die diffusen intrinsischen Ponsgliome und die Gliomatosis cerebri, die neu diagnostiziert wurden und bisher unbehandelt sind, werden aktuell in die HIT-HGG-2013-Studie eingeschlossen.

\section{Inform Register (Individualized Therapy}

For Relapsed Malignancies in Childhood)

In dieses Register können Kinder, Jugendliche und junge Erwachsene von 1-40 Jahren mit einer refraktären, rezidivierten oder progressiven onkologischen Erkrankung, für die keine etablierten kurativen Therapieoptionen bestehen, eingeschlossen werden. Das Register wird an der Universität Heidelberg durchgeführt. Es dient der Etablierung der Logistik, einem individuell auf den Patienten abzielenden Risikomanagement sowie der Etablierung von Zugangswegen zu möglichen zielgerichteten Therapien mittels klinisch relevanter Targets inkl. Einzelheilversuche.

Der Zugewinn an molekularen Erkenntnissen bei einer Vielzahl kindlicher Tumoren ist in den vergangenen Jahren enorm und hat bereits bei einigen Tumoren zu prognostischen Graduierungen und letztendlich Therapieentscheidungen geführt. So kann für Kinder und Erwachsene mit einem malignen Gliom, welches eine DNA-Hypermethylierung im Bereich des MGMT-Promotors (O6-Methylguanin-DNA-Methyltransferase) aufweist und damit zu einer Repression des MGMT-Gens führt, ein gutes Ansprechen auf eine Therapie mit Temozolomid erhofft werden. Auch eine Kodeletion auf den Chromosomen 1p und $19 q$ in IDH-mutierten Gliomen hat eine positive prognostische Wertigkeit.

\section{Merke}

Die genetische Charakterisierung von Hirntumoren hat zu einem neuen Verständnis über die klinische Diversität von scheinbar gleichen Tumoren nach WHO-Standard geführt.

Diese Tatsache spiegelt sich auch in den aktuellen Therapieempfehlungen für Medulloblastome wider: Gegenwärtig werden Medulloblastome WHO-Grad IV nach histologischen Kriterien in 4 Typen unterteilt:
- klassisch

- desmoplastisch/nodulär

- mit extensiver Nodularität

- großzellig/anaplastisch

Demgegenüber steht eine molekularbiologische Klassifikation, die ebenfalls 4 Subtypen unterscheidet:

- WNT-Medulloblastom

- TP53-WT-SHH-Medulloblastom

- TP53-Mut-SHH-Medulloblastom

- Medulloblastom non WNT/non SHH

Das WNT-Medulloblastom umfasst mit ca. $15 \%$ die kleinste Gruppe (inkl. der Medulloblastome, die im Rahmen eines Turcot-Syndroms auftreten). Die Tumoren tragen Mutationen, die zu einer Aktivierung des WNT-Signalweges führen. Prognostisch ist dies die günstigste molekulargenetische Medulloblastomvariante mit einem 5Jahres-Überleben von $>90 \%$.

Das TP53-WT-SHH-Medulloblastom kommt vorwiegend in den Altersgruppen $<3$ und $>15$ Jahren vor und macht ca. $20 \%$ aller Medulloblastome aus (inkl. der Medulloblastome, die im Rahmen eines Gorlin-Syndroms auftreten). Zugrunde liegt eine konstitutive Aktivierung des $\mathrm{SHH}$ Signalweges (sonic hedgehog), z. B. aufgrund von Mutationen bei PTCH1, SMO oder SUFU. Mutationen im TP53-Gen liegen definitionsgemäß nicht vor. Die Art der Mutation innerhalb des SHH-Signalwegs ist dabei prädiktiv für das Ansprechen auf die Behandlung mit SMO-Inhibitoren.

TP53-Mut-SHH-Medulloblastome treten zumeist im Alter zwischen 5 und 15 Jahren auf und tragen ein hohes Risiko für das Vorliegen eines Li-Fraumeni-Syndroms, d. h. einer TP53-Keimbahnmutation. Das Hinzuziehen eines Humangenetikers ist bei Vorliegen eines solchen Tumors indiziert.

Medulloblastome non WNT/non SHH stellen die größte Gruppe dar und gehören weder zur WNT- noch zur SHHGruppe. Die zugrunde liegende Molekularbiologie ist im Wesentlichen noch unverstanden. Anhand von epigenetischen Veränderungen und ihrer Genexpression unterscheidet man aber Gruppe-3- und -4-Tumoren, wobei die Prognose der Gruppe-3-Tumoren besonders schlecht ist.

Eine klare Korrelation zwischen den traditionellen histologischen und den molekularen Medulloblastomvarianten gibt es zwar nicht, jedoch lassen sich die desmoplastischen und die mit extensiver Nodularität fast ausschließlich den SHH-Medulloblastomen zuordnen, wohingegen großzellige/anaplastische Tumoren den Medulloblastomen non WNT/non SHH entsprechen. 


\section{Merke}

Vor allem in Rezidivsituationen stehen nur noch bedingt standardisierte Protokolle zur Verfügung. Häufig ist eine erneute Resektion nicht oder nur mit entsprechenden Einschränkungen für den Patienten möglich, die kumulative Bestrahlungsdosis wurde bereits erreicht und eine konventionelle Polychemotherapie verspricht kein erneutes Ansprechen.

In solchen Fällen müssen alternative Optionen bereitstehen. Eine Option ist die „molecular targeted therapy“, also eine medikamentöse Therapie, die direkt in die Signalwege eingreift, die die Tumorzelle zum Überleben, Proliferieren und Migrieren benötigt. Ziel des InForm-Registers ist es, solche Signalwege ausfindig zu machen. Sollte ein „drugable target“ gefunden werden, so kann mit einem entsprechenden Inhibitor oder einer passenden Kombination ein Therapieversuch unternommen werden. Zumeist verspricht eine Kombinationstherapie bessere Ergebnisse, teilweise auch die metronomische Anwendung von Medikamenten oder die Kombination von Molecular targeted Therapy und konventioneller Polychemotherapie. Ein Beispiel dafür ist das „RIST-Regime“: Es handelt sich um die Anwendung von 4 Medikamenten (Rapamycin, Dasatinib [Sprycel], Temozolomid und Irinotecan), wobei in einer Woche Rapamycin und Dasatinib und in der Folgewoche Irinotecan und Temozolomid verabreicht werden. Rapamycin fungiert als mTorInhibitor, Dasatinib als relativ breit wirksamer Tyrosinkinsaseinhibitor. Irinotecan und Temozolomid zählen zu den klassischen Chemotherapeutika. Diese Therapie verzeichnet vor allem bei Rezidiven von Medulloblastomen oder Glioblastomen zumindest mittelfristige Ansprechraten.

Eine weitere Therapieoption ist die Immuntherapie und in diesem Bereich der Einsatz von sog. Checkpoint-Inhibitoren. Als Checkpoints werden Signalübertragungen bezeichnet, die die natürliche T-Zell-Antwort begrenzen und Autoimmunität verhindern. Durch die Blockade dieser Rezeptoren wie PD-L1 oder CTLA-4 wird eine präexistente CTL-Antwort freigesetzt, die gegen den Tumor wirkt. Als Substanzen werden bei kindlichen Hirntumoren vor allem Nivolumab oder Ipilimumab eingesetzt. Im Rahmen der CA209-908-Studie wurden diese Substanzen an den verschiedensten Hirntumorrezidiven getestet.

Zusammenfassend kann gesagt werden, dass gerade für die Behandlung von Rezidiven weitere Studien nötig sind, um vielversprechende Substanzen zu testen und mehr bzw. bessere Optionen für die Therapie zu haben. Allerdings eröffnen sich genau hier neue Schwierigkeiten, denn schon der deutsche Arzt Abraham Jacobi, der als einer der Gründer der modernen Kinderheilkunde gilt, formulierte die Prämisse: Kinder sind nicht kleine Erwachsene [19].
Pädiatrische Hirntumoren (oder generell Krebserkrankungen), sind eine eigenständige Erkrankung, die getrennt von vergleichbaren Erkrankungen bei Erwachsenen verstanden werden muss. So ist der durchschnittliche „mutational landscape“ bei pädiatrischen Tumoren viel geringer als bei adulten Krebserkrankungen, d. h. es gibt weniger tumorspezifische Proteinveränderungen, sodass es weniger tumorspezifische „drugable targets“ gibt [20]. Die Tatsache, dass es durch die Behandlung zu einer Selektion von Tumorzellen kommt, die nur noch bedingt Ähnlichkeiten mit den initialen Tumorzellen aufweisen, verkompliziert diesen Umstand weiter. So konnte z. B. beim Neuroblastom gezeigt werden, dass ein primäres Neuroblastom sich hauptsächlich durch DNA-Deletionen und Proteinüberexpression auszeichnet, während das behandelte Rezidiv vermehrt Mutationen aufweist [21]. Dies bedeutet: Erkenntnisse, die aus klinischen Studien an Erwachsenen oder gemischten Populationen (Erwachsene/Heranwachsende/Kinder) gewonnen wurden, können nur bedingt bei pädiatrischen Hirntumoren genutzt werden. Generell besteht hier die Frage: Welche Informationen kann man aus den Studien mit adulten Patienten auf Kinder übertragen? Sicherlich sind Pharmakokinetik, Verträglichkeit und Blut-Hirn-Schranken-Passierbarkeit Fragen von allgemeiner Relevanz. Andere wichtige Aspekte müssen hier allerdings differenzierter betrachtet werden: So ist beispielsweise der Wert einer Therapie, die 30 Jahre nach Behandlungsende Sekundärerkrankungen auslösen kann, bei einem 60-jährigen Patienten anders zu bewerten als bei einem 3-Jährigen.

Klinische Studien an Kindern, abgesehen von den potenziellen ethischen Bedenken, haben ihre eigenen logistischen Probleme, allen voran die geringe Fallzahl. Die hier beschriebenen Studienprotokolle der $\mathrm{GPOH}$ sind eine recht erfolgreiche Reaktion darauf, aber auch andere Modifikationen zum klassischen Studienaufbau sind zur Anwendung gekommen [22]. So wurde z. B. das Rolling Six Design entwickelt, welches die Dauer einer pädiatrischen Phase-I-Studie signifikant verkürzt. Auch die integrierte kombinierte Phase-I/II-Studie mit sequenziellen Proof-ofMechanism- und Proof-of-Concept-Stadien, also eine Studie, die zugleich Dosissteigerung und einen direkten klinischen Nutzen adressiert, wird oft als Möglichkeit propagiert, schneller neue therapeutische Ansätze in den klinischen Standard zu integrieren.

Auch in der präklinischen Forschung hat man sich auf die besonderen Eigenheiten der pädiatrischen Hirntumoren eingestellt: Etablierte Zelllinien, die gerade bei Hirntumoren wie dem Glioblastom nur sehr limitiert das Verhalten von Tumorzellen im Patienten widerspiegeln [23], wurden in den letzten Jahren vermehrt durch pädiatrisches Primärmaterial ersetzt, das - durch besondere Kultivierungsbedingungen - viel genauer die Charakteristika des Tumors reflektiert. In den letzten Jahren hat es (nicht nur bei den pädiatrischen Erkrankungen) zudem eine Ver- 
schiebung des Fokus auf einen Therapieansatz gegeben, der älter als Chemo- und Radiotherapie ist, die sog. Immunotherapie [24]. Zwar beschrieb Rudolph Virchow schon 1863 Immunzellinfiltrate ins Krebsgewebe und 1891 zeigte William Coley, dass therapeutische Modulation des Immunsystems Tumorwachstum beeinflussen kann, aber erst in den letzten 15 Jahren wurde dieser Ansatz klinisch nutzbar weiterentwickelt. Check-Point-Inhibitoren und Car-T-Zellen kombinieren systemische Wirkung von Chemotherapie mit der Spezifität eines chirurgischen Eingriffs. Mit wenigen kontrollierbaren Nebenwirkungen (allen voran das mögliche Auslösen eines Cytokinsturms) und dem Potenzial, den Tumor zu eradizieren und eine nachhaltige Immunität gegen Rezidive aufzubauen, wird dieser Therapieansatz auch in Zukunft bei pädiatrischen Hirntumoren vermehrt eine Rolle spielen.

\section{Strahlentherapie}

Die Strahlentherapie von ZNS-Tumoren im Kindesalter ist hocheffektiv und verbessert in vielen Fällen nicht nur die lokale Kontrolle, sondern auch das Gesamtüberleben insbesondere nach inkompletter Resektion. Wegen der Strahlensensibilität des kindlichen Gewebes ist vor dem Einsatz der Strahlentherapie aber immer eine Abschätzung des Nutzen-Risiko-Profils durchzuführen und möglichst Rücksprache mit den Studienzentralen zu halten. Die Behandlung erfolgt i.d.R. in Studienprotokollen, um bei diesen seltenen Erkrankungen eine Bündelung von Erfahrung zu erreichen. Neben der Tumorkontrolle haben die Minimierung von Nebenwirkungen und damit verbundenen Einschränkungen der Lebensqualität höchste Priorität. Neurokognitive Spätfolgen sowie Zweitmalignomraten sollen reduziert werden, indem die zu bestrahlenden Zielvolumina und die zu applizierenden Strahlendosen möglichst klein gehalten werden, und indem durch die Anwendung hochtechnologischer Bestrahlungsverfahren die Strahlenbelastung von nicht beteiligten ZNS-Arealen minimiert wird [25].

Bei der Strahlentherapie von ZNS-Tumoren ist auf eine weitgehende Immobilisation der Patienten während der Bestrahlung zu achten (Maskensysteme, individuelle Lagerungsschalen, wenn nötig Narkose). Eine 3-dimensionale computergestützte Bestrahlungsplanung unter Einbeziehung der präoperativen Bildgebung und des Operationsberichts zur zuverlässigen Erfassung der Tumorregion (resp. ehemaligen Tumorregion) mit Sicherheitssaum und adäquater Schonung von Normalgewebe ist obligat.
Cave

Zusätzlich ist bei Bestrahlungen im Kindesalter wegen der Strahlenempfindlichkeit der Wachstumsfugen zu beachten, dass eine möglichst symmetrische Bestrahlung von Knochenstrukturen zur Vermeidung von Wachstumsdeformitäten vor allem im Bereich der Wirbelsäule erfolgen muss.

Intrakraniell können Strahlendosen von über 60 Gy appliziert werden. Es sind hierbei jedoch Risikoorgane zu beachten und ggf. auszusparen, die eine geringere Toleranzdosis aufweisen (z.B. Chiasma opticum, Sehnerven, Hypophyse, Hirnstamm).

Cave

Am Rückenmark sollte die Strahlendosis wegen erhöhter Strahlenempfindlichkeit 50 Gy nicht übersteigen, um das Risiko einer Myelopathie gering zu halten.

Das Risiko für radiogene Spätfolgen steigt generell mit dem bestrahlten Volumen an sowie auch mit der Höhe der täglichen Einzeldosis und auch mit der kumulativen Gesamtdosis. In der Regel werden eher kleine tägliche Strahlendosen von 1,8-2 Gy appliziert; manchmal auch besonders geringe Einzeldosen $2 \times$ pro Tag (Hyperfraktionierung). Bei kurativen Behandlungsansätzen resultiert daraus im Allgemeinen eine Bestrahlungsdauer von 4-7 Wochen.

Es kommen verschiedene Techniken (z.B. 3D-geplante Techniken mittels CT/MRT/ggf. PET-CT, Stereotaxie, intensitätsmodulierte Strahlentherapie, Protonentherapie) zum Einsatz - immer mit dem Ziel, im Tumorbereich eine möglichst homogene Dosisverteilung zu erreichen, bei optimaler Schonung des umgebenden gesunden Gewebes.

Das Gehirn entwickelt sich sehr stark innerhalb der ersten 3 Lebensjahre, während sich nachfolgend die Hirnreifung deutlich verlangsamt und nach dem 6 . Lebensjahr weitgehend abgeschlossen ist. Die Myelinisierung ist in der Pubertät vollständig. Dementsprechend sind radiogen bedingte, zerebrale Beeinträchtigungen vor allem bei Bestrahlungen im sehr jungen Kindesalter (bis 3 Jahre) zu erwarten [26].

\section{Merke}

Bei Säuglingen und Kleinkindern unter 3 Jahren wird daher die Bestrahlung des ZNS aufgrund des hohen Nebenwirkungsrisikos wenn möglich zurückgestellt bzw. die Zeit bis zur nötigen Strahlentherapie durch eine Chemotherapie verlängert. 
Wenn zeitnah verfügbar, sollte bei Kindern und Jugendlichen eine Protonentherapie evaluiert werden. Die antineoplastische Wirksamkeit von Photonen und Protonen ist vergleichbar. Protonen zeichnen sich jedoch gegenüber den herkömmlicherweise verwendeten Photonen durch spezielle physikalische Eigenschaften aus: Aufgrund unterschiedlichen Absorptionsverhaltens im Gewebe kann mittels Protonen eine sehr genaue Dosismodellierung erfolgen, mit Erzeugung starker Dosisgradienten an definierter Stelle. Dadurch ist eine Reduktion der Strahlenbelastung im gesunden Gewebe möglich, weil die bei der konventionellen Strahlentherapie auftretende Niedrigdosisbelastung auch von weiter entfernt gelegenen gesunden Gewebestrukturen teilweise eliminiert werden kann. Insbesondere bei Kindern und jungen Erwachsenen verspricht die Protonentherapie eine Reduktion von mittel- und langfristigen Nebenwirkungen [27]. Die Protonentherapie ist eine hochkomplexe strahlentherapeutische Behandlungsform, die nur in spezialisierten Zentren durch erfahrene Radioonkologen durchgeführt wird und bisher nur in wenigen Instituten mit entsprechender aufwendiger technischer Ausstattung verfügbar ist.

In der Regel wird die Strahlentherapie heutzutage gut toleriert, da durch die Anwendung von modernen Techniken und supportiven Maßnahmen die Rate an Akutund Spätnebenwirkungen bereits deutlich reduziert werden konnte. Schwerwiegende oder vital bedrohliche Nebenwirkungen treten bei der Strahlentherapie äußerst selten auf, am ehesten noch im Zusammenhang mit der Applikation weiterer Tumortherapien (z. B. Zytopenie).

\section{Merke \\ Nach Abschluss der ZNS-Bestrahlung kann es nach 4-8 Wochen zu einem Somnolenz-Syndrom kommen. Es äußert sich in Benommenheit, Apathie, Übelkeit, Anorexie und/oder Irritabilität. Das Somnolenz-Syn- drom ist passager, die Gabe von Kortikosteroiden kann die Erholung beschleunigen.}

Die Inzidenz strahleninduzierter Nekrosen im Bereich des ZNS wird nach 50-60 Gy mit 0,1-5\% angegeben und ist abhängig von der Größe und Lokalisation des Zielvolumens, der Fraktionierung und dem Alter des Patienten. Kleinere Nekrosen im Zielvolumen (d.h. im Tumorbereich) sind klinisch meist wenig bedeutsam. Schwerwiegende Leukenzephalopathien (mit klinischen Symptomen wie Lethargie, Anfälle, Spastik, Paresen, Ataxie, Abfall der neurokognitiven Leistungen bis hin zur Demenz) sind inzwischen vermeidbar. Derartig gravierende radiogene Nebenwirkungen waren in früherer Zeit gelegentlich zu beobachten, bei großvolumigen und recht hochdosierten ZNS-Bestrahlungen von Kleinkindern, sowie vor allem bei gleichzeitiger intravenöser und intrathekaler Gabe von Methotrexat. Histologisch kam es in diesen
Fällen zu einer multifokalen Nekrose der weißen Substanz. Durch vorsichtigere Indikationsstellung sowie moderne Dosis- und Volumenkonzepte sind derartige Nebenwirkungen in der modernen Strahlentherapie nicht mehr zu erwarten. Die weitere Entwicklung muss zum Ziel haben, auch geringere Nebenwirkungen (z. B. leichtere intellektuelle Defizite oder eingeschränkte Berufswahlmöglichkeiten bei ZNS-bestrahlten Kindern und Jugendlichen) zu minimieren.

Die Zuordnung von langfristigen Nebenwirkungen nach der Behandlung von ZNS-Tumoren ist allerdings schwierig: Sowohl die Erkrankung selbst als auch die operative und zytostatische Therapie tragen neben der Bestrahlung zu neurokognitiven Defiziten bei.

Daten zu Zweittumoren nach Behandlung von Tumoren des ZNS sind aufgrund fehlender systematischer Erfassungen und unterschiedlicher Therapiekonzepte (Operation, Chemo- und Strahlentherapie) schwierig zu interpretieren. Langzeitüberlebende scheinen ein bis zu 10fach erhöhtes Risiko im Vergleich zur Normalbevölkerung aufzuweisen [28]. Zu den strahlentherapieinduzierten Tumoren gehören Meningeome, maligne Astrozytome oder intrakranielle Fibrosarkome. Die kumulative Inzidenz beträgt nach 20 Jahren circa 2\%. Das Alter scheint einen zusätzlichen prognostischen Faktor zu bilden. Durch den Einsatz der Protonentherapie besteht die Aussicht, dass das Zweittumorrisiko vermindert werden könnte [27].

\section{Palliativmedizin}

Seit einigen Jahren besteht in Deutschland der Rechtsanspruch auf eine ambulante Palliativversorgung. Dies gilt uneingeschränkt auch für Kinder und Jugendliche. In Baden-Württemberg wird seit Anfang 2016 durch insgesamt 5 SAPV-Teams (Heidelberg/Mannheim, Stuttgart, Tübingen, Freiburg, Ulm/Ravensburg) eine entsprechende flächendeckende Versorgung gewährleistet und durch die Krankenkassen finanziert. Aufgrund der - verglichen mit der Erwachsenenmedizin - geringen Patientendichte sind somit sehr große Versorgungsgebiete (Durchmesser $>100 \mathrm{~km}$ ) entstanden.

Beispielhaft versorgt das SAPV-Team in Ulm und Ravensburg „Pallikjur“ pro Quartal ca. 25 PatientInnen in einem Bereich zwischen Schwäbisch Hall und dem Bodensee. Das Patientenkollektiv setzt sich zu ca. 50\% aus onkologischen, klassischen PalliativpatientInnen und zur anderen Hälfte aus schwerst mehrfach behinderten Patienten mit nicht heilbaren Erkrankungen und unklarer zeitlicher Prognose zusammen.

Intendiert wird die möglichst vollständige Beibehaltung des häuslichen Umfelds der Kinder und Jugendlichen. Zu den Kernaufgaben gehören: 


\section{FALLBEISPIEL}

\section{Primär metastasiertes Medulloblastom}

Mädchen, 12 Jahre, Diagnose: primär metastasiertes Medulloblastom; wohnhaft ca. 120 km entfernt von der betreuenden Kinderonkologie.

Nach der Erstdiagnose klassische multimodale onkologische Therapie mit Operation, Radiatio und Zytostatika. Mit Rezidivdiagnose 2,5 Jahre später wurde eine ambulante, teils tagesstationäre Second-LineTherapie durchgeführt. Es kam ca. 1 Jahr darauf zum Tumorprogress, sodass unter palliativer Maßgabe eine orale zytostatische Therapie mit zusätzlicher intrathekaler MTX-Applikation im Monatsrhythmus begonnen wurde. 3 Monate danach erfolgte nach fast 4 Jahren intensiver onkologischer Betreuung die Aufnahme in die ambulante Palliativversorgung. Seitdem musste sich die Patientin nur noch im Einzelfall im Krankenhaus vorstellen und wird fast ausschließlich zu Hause versorgt. Diese Versorgung umfasst die Therapiesteuerung, die Rezeptierung und Organisation der individuellen Zubereitung der Zytostatika, die Supportivtherapie, invasive Maßnahmen wie Blutentnahmen und Rickham-Punktionen im häuslichen Umfeld sowie die medizinische Notfallversorgung. Nicht zu vergessen ist die Unterstützung der Familie in der Planung und Durchführung von Urlauben und Rehabilitationsmaßnahmen. Die Patientin besucht weiterhin die Schule und hat einen - im Rahmen der Möglichkeiten - normalen Alltag.

- Symptomkontrolle

- adäquate Versorgung mit Medikamenten und Hilfsmitteln

- qualifizierte sozialrechtliche Betreuung

- Beratung bezüglich des Vorgehens in medizinischen Notfallsituationen

- kontinuierlicher ärztlicher Rufdienst

Regelmäßig werden auch PatientInnen mit Hirntumoren betreut. Hier liegt die besondere Herausforderung im Management der oft progredienten neurologischen Symptome (z. B. Therapie von Krampfanfällen) sowie der klinischen Probleme, die mit der häufig begleitenden, zunehmenden Einschränkung der Vigilanz einhergehen.

Ein wichtiger Aspekt ist nach unserer Ansicht die Möglichkeit einer unterstützenden oder vollständigen Versorgung durch uns, selbst wenn die PatientInnen z. B. in Re- zidivsituationen noch experimentelle (palliative) zytostatische Therapien erhalten. Die Teams verfügen üblicherweise über die onkologische Expertise, solche Therapien zu verabreichen, zu monitoren und erforderliche supportive Maßnahmen (Symptomkontrolle bei Schmerzen, epileptischen Anfällen, therapieassoziierte Übelkeit, Obstipation oder Diarrhö) zu initiieren. Ziel ist, eine möglichst hohe Lebensqualität sicherzustellen, um insbesondere ambulante Krankenhausbesuche mit häufig langen Anfahrtswegen zu vermeiden. Um eventuelle Vorbehalte seitens der PatientInnen und ihrer Eltern zu vermeiden, sollte der/die behandelnde/r Onkologln in einer entsprechenden klinischen Situation frühzeitig das zuständige Palliativteam einbeziehen.

\section{Wichtige Links}

https://www.uniklinik-ulm.de/kinder-und-jugendmedizin/sektionen-ambulanzen-und-arbeitsbereiche/palliativmedizin.html

https://kinder-palliativ-landesstelle.de/spezialisierte-ambulante-palliativversorgung-sapv/

\section{KERNAUSSAGEN}

- Neben den Leukämien sind kindliche Hirntumoren die häufigsten Neoplasien im Kindesalter. Nicht selten werden diese erst spät diagnostiziert. Zeichen des erhöhten intrakraniellen Drucks bei Kindern sind Nüchternerbrechen, perzentilenkreuzendes Kopfwachstum, gespannte Fontanelle, Irritabilität und epileptische Anfälle.

- Für die meisten kindlichen Hirntumoren ist das Ausmaß der mikrochirurgischen Tumorentfernung, sofern dies mit einem geringen Risiko für funktionelle Defizite möglich ist, ein entscheidender prognostischer Faktor.

- Die molekularen Signaturen von kindlichen Hirntumoren werden für Therapieentscheidungen und die Prognoseabschätzung immer mehr an Bedeutung gewinnen.

- Die Strahlentherapie bei Kindern unter 3 Jahren sollte wegen des Risikos für neurokognitive Defizite, Wachstumsretardierung und Zweitmalignome nur in Ausnahmefällen erfolgen.

- Die ambulante Palliativversorgung kann und sollte frühzeitig in die onkologische Therapie eingebunden werden. 


\section{Interessenkonflikt}

Erklärung zu finanziellen Interessen

Forschungsförderung erhalten: nein; Honorar/geldwerten Vorteil für Referententätigkeit erhalten: nein; Bezahlter Berater/interner Schulungsreferent/Gehaltsempfänger: nein; Patent/Geschäftsanteile/Aktien (Autor/Partner, Ehepartner, Kinder) an Firma (Nicht-Sponsor der Veranstaltung): nein; Patent/Geschäftsanteile/Aktien (Autor/Partner, Ehepartner, Kinder) an Firma (Sponsor der Veranstaltung): nein.

Erklärung zu nichtfinanziellen Interessen

Die Autorinnen/Autoren geben an, dass kein Interessenkonflikt besteht.

\section{Autorinnen/Autoren}

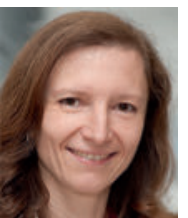

\section{Aurelia Peraud}

Prof. Dr. med.; 1987-1993 Studium der Humanmedizin an der Medizinischen Hochschule Hannover, 1993-2001 Facharztausbildung Neurochirurgie an der LMU München, 19961998 Postdoctoral Fellow am Institut für Neuropathologie, Universitätsspital Zürich,

Schweiz, und an der Unit of Molecular Pathology der International Agency for Research on Cancer in Lyon, Frankreich, 2002-2003 Fellowship in Pediatric Neurosurgery am Hospital for Sick Children in Toronto, Kanada, 2005 Habilitation, 2006 Spina bifida Fellowship am Children's Memorial Hospital in Chicago, USA. Seit 2007 Leiterin des Bereichs Kinderneurochirurgie an der LMU München. Seit 2018 W3-Professur für Pädiatrische Neurochirurgie am Universitätsklinikum Ulm. Schwerpunkte: Mikrochirurgie bei Hirn- und Rückenmarkstumoren, Spina bifida, Epilepsiechirurgie, Hydrocephalus, Schädeldachmissbildungen, kindliches Schädel-Hirn-Trauma.

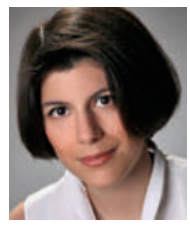

\section{Marie Schuler-Ortoli}

Dr. med.; 2000-2006 Studium der Humanmedizin an der Medizinischen Universität René Descartes Paris V in Paris, Frankreich, 20072016 Facharztausbildung Neurochirurgie am Universitätsklinikum Ulm und am Bezirkskrankenhaus Günzburg, 2017 Promotion. Seit 2018 Oberärztin in der Sektion Pädiatrische Neurochirurgie am Universitätsklinikum Ulm.

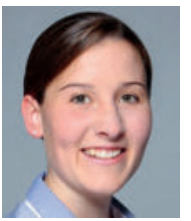

\section{Jana Stursberg}

Dr. med.; 2005-2012 Studium der Humanmedizin an der Universität Ulm, 2012-2019 Facharztausbildung Pädiatrie. Seit 2019 in Weiterbildung pädiatrische Hämatologie und Onkologie.

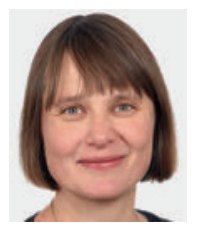

\section{Wiebke Schlötzer}

Dr. med.; 1996-2003 Medizinstudium Universität Leipzig und Ulm, 2004-2005 ÄiP bzw. Assistenzärztin Innere Medizin KKH Günzburg. Seit 2010 Oberärztin an der Klinik für Diagnostische und Interventionelle Radiologie Universitätsklinikum Ulm. 2010 Fachärztin für Diagnostische Radiologie. 2013 Erwerb Schwerpunkt Neuroradiologie. Schwerpunkte: diagnostische Neuroradiologie, pädiatrische Neuroradiologie.

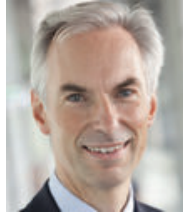

\section{Meinrad Beer}

Prof. Dr. med.; 1988-1995 Studium der Humanmedizin an der Universität Regensburg und Würzburg, 1995-2003 Facharztausbildung Diagnostische Radiologie an der Universität Würzburg, 2004 Habilitation. Seit 2003 Oberarzt und seit 2007 leitender Oberarzt am Institut für Röntgendiagnostik der Universität Würzburg. 2006 Schwerpunktbezeichnung Kinderradiologie, 2009 Universitätsprofessor an der Universität Würzburg, Universitätsprofessor an der Universität Graz, Österreich. Seit 2013 Ärztlicher Direktor der Klinik für Diagnostische und Interventionelle Radiologie der Universitätsklinik Ulm.

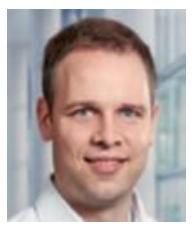

\section{Stephan Linus Bartholomä}

Dr. med.; 2002-2008 Studium der Humanmedizin an der Universität Ulm. 2008-2015 Facharztausbildung Kinder- und Jugendmedizin am Universitätsklinikum Ulm. 2016 Koordinator im Pädiatrischen Palliativteam „Pallikjur“, 2017 Schwerpunktbezeichnung Pädiatrische Hämatologie und Onkologie. Seit 2018 Oberarzt an der Klinik für Kinder- und Jugendmedizin am Universitätsklinikum Ulm. Klinische Schwerpunkte: Onkologie, Palliativmedizin.

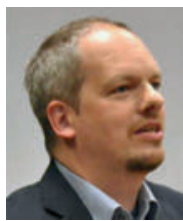

\section{Mike-Andrew Westhoff}

Dr. 1995-1999 Studium der Genetik an der University of Glasgow, Schottland, 1999-2002 Ph. D. in Molecular Pathology and Cancer Sciences am Beatson Institute for Cancer Research Glasgow, Schottland. Ab 2002 Post-Doc und seit 2011 Co-Gruppenleiter Forschungslabor Universitätsklinik für Kinder- und Jugendmedizin, Universitätsklinikum Ulm. Schwerpunkte: Apoptose, Hirntumoren und Kombinationstherapien.

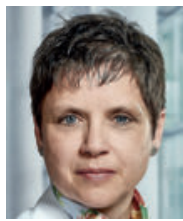

Birgitta Welte

Dr. med.; 1987-1995 Studium der Humanmedizin an der Universität Tübingen, 1995-1997 Assistenzärztin Innere Medizin, 1997-2002 Facharztausbildung Strahlentherapie am Universitätsklinikum Ulm. Seit 2002 Oberärztin in der Klinik für Strahlentherapie und Radioonkologie des Universitätsklinikum Ulm. Zusatzbezeichnung: Palliativmedizin; Schwerpunkt: pädiatrische Radioonkologie.

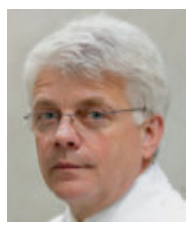

\section{Thomas Wiegel}

Prof. Dr. med.; 1996-2005 Leitender Oberarzt, Klinik für Strahlentherapie, Universitätsklinikum Benjamin Franklin, Charité, Berlin. Seit 2005 Ärztlicher Direktor der Klinik für Strahlentherapie und Radioonkologie des Universitätsklinikums Ulm. 


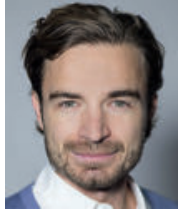

\section{Wolfgang Loichinger}

1998-2005 Studium der Humanmedizin an der Universität Ulm, 2005-2011 Ausbildung zum Facharzt für Kinder- und Jugendmedizin in Heidenheim und Ulm, 2011 Facharzt für Kinder- und Jugendmedizin, 2014 Schwerpunktbezeichnung Pädiatrische Hämatologie und Onkologie, 2017 Zusatzbezeichnung Palliativmedizin. Klinische Schwerpunkte: Onkologie, benigne Hämatologie, Palliativmedizin, Ultraschalldiagnostik (DEGUM-Ausbilder).

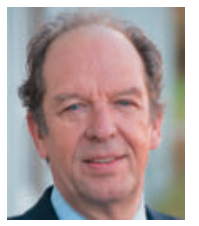

\section{Klaus-Michael Debatin}

Prof. Dr. med.; 1971-1978 Medizinstudium Universitäten Ulm, Freiburg, Heidelberg, 1979 Promotion, Universität Heidelberg. 1990 Habilitation im Fach Kinderheilkunde, Universität Heidelberg. 1990-1991 DFG Forschungsstipendium und „Fogarty Fellow“ National Cancer Institute Bethesda, USA, 1991-1994 Heisenberg Stipendiat der DFG, National Cancer Institute, USA - DKFZ, Heidelberg Hôpital Necker, Paris. 1994-1997 Leiter der Sektion Hämatologie/Onkologie der Universitäts-Kinderklinik Heidelberg. 1997 Visiting Professor, St. Jude Children's Research Hospital, Memphis. 1995-2004 Leiter der Abteilung Molekulare Onkologie/Pädiatrie am Deutschen Krebsforschungszentrum Heidelberg. 2002-2004 Prodekan der Medizinischen Fakultät der Universität Ulm, 2003-2005 Vorsitzender des Wissenschaftskomitees der Medizinischen Fakultät, Universität Ulm. 20042010 Dekan der Medizinischen Fakultät der Universität Ulm, 2010-2013 Vizepräsident für Medizin der Universität UIm, 2010-2013 Stellvertretender Leitender Ärztlicher Direktor des Universitätsklinikums Ulm, 2013-2015 Leitender Ärztlicher Direktor des Universitätsklinikums Ulm. Seit 1997 Ärztlicher Direktor der Universitätsklinik für Kinder- und Jugendmedizin Ulm. Seit 2015 Vizepräsident für Medizin der Universität Ulm. Schwerpunkte: Pädiatrische Hämatologie/Onkologie, Molekulare Onkologie, Apoptose, Experimentelle Therapien.

\section{Korrespondenzadresse}

Prof. Dr. med. Aurelia Peraud

Sektion Pädiatrische Neurochirurgie, Klinik für Neurochirugie Universitätsklinikum Ulm

Albert-Einstein-Allee 23

89081 Ulm

aurelia.peraud@uniklinik-ulm.de

Wissenschaftlich verantwortlich gemäß Zertifizierungsbestimmungen

Wissenschaftlich verantwortlich gemäß Zertifizierungsbestimmungen für diesen Beitrag ist Prof. Dr. med. Aurelia Peraud, Ulm.

\section{Zitierweise für diesen Artikel}

Onkologie up2date 2020; 2: 327-345.

doi:10.1055/a-1261-6247

Dieser Beitrag ist eine aktualisierte Version des Artikels: Peraud A, Schuler-Ortoli M, Stursberg J, Schlötzer W, Beer M, Bartholomä S, Westhoff MA, Welte B, Wiegel T, Loichinger W, Debatin KM. Aktuelle Behandlungskonzepte für Hirntumoren im Kindes- und Jugendalter. Pädiatrie up2date 2020; 15: 55-73.
Literatur

[1] Kaatsch P, Spix C, Grabow D, Becker C. Annual Report 2017 German Childhood Cancer Registry GCCR. Mainz: Institute of Medical Biostatistics, Epidemiology and Informatics (IMBEI) at the University Medical Center of the Johannes Gutenberg University Mainz; 2017

[2] Rutkowski S, Trollmann R, Korinthenberg R et al. S1-Leitlinie 025/022. AWMF; 2016. Im Internet: www.awmf.org/uploads/ tx_szleitlinien/025-022I_S1_ZNS-Tumoren_Kinder_Jugendliche_2016-09.pdf; Stand:16.01.2020

[3] Timmermann B. S1-Leitlinie 025/025. AWMF; 2018. Im Internet: www.awmf.org/uploads/tx_szleitlinien/025-025I_S1_Epen dymome-Kinder-Jugendliche_2018-04.pdf; Stand 16.01.2020

[4] Rutkowski S. S1-Leitlinie 025/009. AWMF; 2018. Im Internet: www.awmf.org/uploads/tx_szleitlinien/025-009l_S1_Medulloblastom-Kinder-Jugendliche_2018-04.pdf; Stand 16.01.2020

[5] Warren KE, Vezina G, Poussaint TY et al. Response assessment in medulloblastoma and leptomeningeal seeding tumors: recommendations from the Response Assessment in Pediatric Neuro-Oncology committee. Neuro Oncol 2018; 20: 13-23. doi:10.1093/neuonc/nox087

[6] Ellingson BM, Bendszus M, Boxerman J et al. Consensus recommendations for a standardized Brain Tumor Imaging Protocol in clinical trials. Neuro Oncol 2015; 17: 1188-1198. doi:10.1093/neuonc/nov095

[7] Kralik SF, Taha A, Kamer AP et al. Diffusion imaging for tumor grading of supratentorial brain tumors in the first year of life. AJNR Am J Neuroradiol 2014; 35: 815-823. doi:10.3174/ajnr. A3757

[8] Rumboldt Z, Camacho DL, Lake D et al. Apparent diffusion coefficients for differentiation of cerebellar tumors in children. AJNR Am J Neuroradiol 2006; 27: 1362-1369

[9] Jaremko JL, Jans LB, Coleman LT et al. Value and limitations of diffusion-weighted imaging in grading and diagnosis of pediatric posterior fossa tumors. AJNR Am J Neuroradiol 2010; 31: 1613-1616. doi:10.3174/ajnr.A2155

[10] Harreld JH, Mohammed N, Goldsberry G et al. Postoperative intraspinal subdural collections after pediatric posterior fossa tumor resection: incidence, imaging, and clinical features. AJNR Am J Neuroradiol 2015; 36: 993-999. doi:10.3174/ajnr. A4221

[11] Koral K, Zhang S, Gargan L et al. Diffusion MRI improves the accuracy of preoperative diagnosis of common pediatric cerebellar tumors among reviewers with different experience levels. AJNR Am J Neuroradiol 2013; 34: 2360-2365. doi:10.3174/ajnr.A3596

[12] Lobel U, Sedlacik J, Reddick WE et al. Quantitative diffusionweighted and dynamic susceptibility-weighted contrast-enhanced perfusion MR imaging analysis of T2 hypointense lesion components in pediatric diffuse intrinsic pontine glioma. AJNR Am J Neuroradiol 2011; 32: 315-322. doi:10.3174/ajnr. A2277

[13] Prager AJ, Martinez N, Beal K et al. Diffusion and perfusion MR to differentiate treatment-related changes including pseudoprogression from recurrent tumors in high-grade gliomas with histopathologic evidence. AJNR Am J Neuroradiol 2015; 36: 877-885. doi:10.3174/ajnr.A4218

[14] Warmuth-Metz M, Kuhl J, Krauss J et al. Subdural enhancement on postoperative spinal MRI after resection of posterior cranial fossa tumours. Neuroradiology 2004; 46: 219223. doi:10.1007/s00234-003-1158-y

[15] Foster MT, Harishchandra LS, Mallucci C. Pediatric Central Nervous System Tumors: State-of-the-Art and Debated Aspects. Front Pediatr 2018; 6: 309. doi:10.3389/fped.2018.00309 
[16] Balogun JA, Rutka JT. Surgery of Intracranial Gliomas in Children. Prog Neurol Surg 2018; 30: 204-217. doi:10.1159/ 000464437

[17] Thompson EM, Hielscher T, Bouffet E et al. Prognostic value of medulloblastoma extent of resection after accounting for molecular subgroup: a retrospective integrated clinical and molecular analysis. Lancet Oncol 2016; 17: 484-495. doi:10.1016/s1470-2045(15)00581-1

[18] Kim JH, Phi JH, Lee JY et al. Surgical Outcomes of Thalamic Tumors in Children: The Importance of Diffusion Tensor Imaging, Neuro-Navigation and Intraoperative Neurophysiological Monitoring. Brain Tumor Res Treat 2018; 6: 60-67. doi:10.14791/btrt.2018.6.e14

[19] Westhoff MA, Marschall N, Grunert M et al. Cell death-based treatment of childhood cancer. Cell Death Dis 2018; 9: 116. doi:10.1038/s41419-017-0062-z

[20] Gröbner SN, Worst BC, Weischenfeldt ] et al. The landscape of genomic alterations across childhood cancers. Nature 2018; 555: 321-327. doi:10.1038/nature25480

[21] Fletcher JI, Ziegler DS, Trahair TN et al. Too many targets, not enough patients: rethinking neuroblastoma clinical trials. Nat Rev Cancer 2018; 18: 389-400. doi:10.1038/s41568-0180003-x

[22] Doussau A, Thiébaut R, Geoerger B et al. A new approach to integrate toxicity grade and repeated treatment cycles in the analysis and reporting of phase I dose-finding trials. Ann Oncol 2015; 26: 422-428. doi:10.1093/annonc/mdu523

[23] Schneider M, Ströbele S, Nonnenmacher L et al. A paired comparison between glioblastoma "stem cells" and differentiated cells. Int J Cancer 2016; 138: 1709-1718. doi:10.1002/ ijc. 29908
[24] Burdach SEG, Westhoff MA, Steinhauser MF et al. Precision medicine in pediatric oncology. Mol Cell Pediatr 2018; 5: 6. doi:10.1186/s40348-018-0084-3

[25] Eaton BR, MacDonald SM, Yock TI et al. Secondary Malignancy Risk Following Proton Radiation Therapy. Front Oncol 2015; 5: 261. doi:10.3389/fonc.2015.00261

[26] Goldsby RE, Liu Q, Nathan PC et al. Late-occurring neurologic sequelae in adult survivors of childhood acute lymphoblastic leukemia: a report from the Childhood Cancer Survivor Study. J Clin Oncol 2010; 28: 324-331. doi:10.1200/JCO.2009.22. 5060

[27] Moteabbed M, Yock TI, Paganetti H. The risk of radiation-induced second cancers in the high to medium dose region: a comparison between passive and scanned proton therapy, IMRT and VMAT for pediatric patients with brain tumors. Phys Med Biol 2014; 59: 2883-2899. doi:10.1088/0031-9155/59/ $12 / 2883$

[28] Turcotte LM, Neglia JP, Reulen RC et al. Risk, Risk Factors, and Surveillance of Subsequent Malignant Neoplasms in Survivors of Childhood Cancer: A Review. J Clin Oncol 2018; 36: 2145 2152. doi:10.1200/JCO.2017.76.7764

Bibliografie

Onkologie up2date 2020; 2: 327-345

DOI 10.1055/a-1261-6247

ISSN 2626-6628

(c) 2020. Thieme. All rights reserved. Georg Thieme Verlag KG, Rüdigerstraße 14, 70469 Stuttgart, Germany 


\section{Punkte sammeln auf CME.thieme.de}

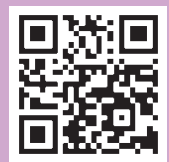

Diese Fortbildungseinheit ist in der Regel 12 Monate online für die Teilnahme verfügbar.

Den genauen Einsendeschluss finden Sie unter https://cme.thieme.de.

Sollten Sie Fragen zur Online-Teilnahme haben, finden Sie unter https://cme.thieme.de/hilfe

eine ausführliche Anleitung. Wir wünschen viel Erfolg beim Beantworten

der Fragen!

Unter https://eref.thieme.de/CXFQ1R7 oder über den QR-Code kommen Sie direkt zur Startseite des Wissenstests.

VNR 2760512020158720074

\section{Frage 1}

Was gehört nicht zu den Alarmsymptomen für ZNS-Tumoren?

A okzipitale Kopfschmerzen

B Nüchternerbrechen

C Kopfschmerzen, die im Liegen zunehmen und/oder den Nachtschlaf stören

D Synkopen

E perzentilenkreuzender Kopfumfang oder vorgewölbte Fontanelle beim Säugling

\section{Frage 2}

Tumoren des zentralen Nervensystems machen im Kindesalter wie viel Prozent der Krebsfälle aus?
A $<2 \%$
B ca. $5 \%$
C ca. $10 \%$
D ca. $25 \%$
E $>33 \%$

\section{Frage 3}

Eine der folgenden Aussagen zu subduralen Effusionen ist falsch. Welche?

A Sie können leicht mit Metastasen verwechselt werden.

B Sie treten gehäuft nach Eingriffen in der hinteren Schädelgrube auf.

C Sie werden auch als unspezifisches subdurales Enhancement bezeichnet.

D Sie weisen i. d. R. eine vollständige Rückbildung innerhalb von wenigen Wochen auf.

E Sie zeigen im MRT typische lobulierte intraspinale Formationen.

\section{Frage 4}

Welches Ziel verfolgt die operative Tumorentfernung bei kindlichen Hirntumoren nicht?
A Symptomkontrolle
B weitgehend komplette Tumorresektion
C Gewebegewinnung für die neuropathologische Diagnostik
D Radikalität steht vor Funktionserhalt
E Entfernung des epileptogenen Fokus

\section{Frage 5}

Eine Radiotherapie bei kindlichem Gehirntumor ...

A muss immer in Narkose durchgeführt werden.

B darf maximal mit 40 Gy erfolgen.

C verbessert das Gesamtüberleben i. Allg. nicht.

D ist bei Kleinkindern keine fest etablierte Standardbehandlung und sollte nur in Ausnahmefällen erfolgen.

E sollte mit Photonen erfolgen, da diese bei gleicher Wirksamkeit wie Protonen günstigere physikalische Eigenschaften aufweisen.

\section{Frage 6}

Was ist keine Therapieoption in der Rezidivsituation bei Hirntumoren?
A Molecular Targeted Therapy
B Immuntherapie
C Radiatio
D chirurgische Therapie
E Magnetresonanztherapie

\section{Frage 7}

Welche Aussage ist falsch?

A Geringe Fallzahlen sind ein Problem bei der Evaluierung neuer Therapieansätze für pädiatrische Tumoren.

B Pädiatrische Tumoren weisen i.d.R. wenig Mutationen auf.

C Informationen aus klinischen Studien mit Erwachsenen sind auch für pädiatrische Patienten von Nutzen.

D Car-T-Zellen sind bei Kindern aufgrund des nicht ausgereiften Immunsystems nicht einsetzbar.

E Chemotherapie wird vor allem zur Reduktion der Bestrahlungsdosis eingesetzt.

\section{Frage 8}

Welches ist der häufigste Hirntumor des Kindesalters?
A das Kraniopharyngeom
B das Medulloblastom
C das Pineoblastom
D das niedermaligne Gliom
E primitive neuroektodermale Tumoren 


\section{Punkte sammeln auf CME.thieme.de}

\section{Fortsetzung $\ldots$}

\section{Frage 9}

Welche Aussage ist falsch?

A Das gelegentlich einige Wochen nach ZNS-Bestrahlung auftretende Somnolenz-Syndrom ist passager.

B Bei kurativer Intention dauert die Strahlentherapie eines ZNSTumors durchschnittlich 6-7 Wochen.

C Großvolumige Bestrahlungen, z. B. des gesamten kindlichen Neurokraniums, ergeben trotz eher geringer Strahlendosis relativ ausgeprägte neurokognitive Beeinträchtigungen.

D Eine Strahlentherapie im ZNS-Bereich wird im Hinblick auf Spätfolgen umso besser vertragen, je jünger das Kind ist.

E Bei der Bestrahlungsplanung kindlicher Hirntumoren werden heute regelmäßig zumindest ein CT und MRT herangezogen.

\section{Frage 10}

Zu den primären Zielen einer palliativmedizinischen Versorgung von pädiatrischen Patienten gehört welcher der folgenden Punkte nicht?
A Symptomkontrolle
B adäquate Versorgung mit Medikamenten und Hilfsmitteln
C Kostenersparnis/Ressourcenschonung
D Beratung bezüglich des Vorgehens in medizinischen Notfall- situationen
E kontinuierlicher ärztlicher Rufdienst 